\title{
Autonomy Loss, Privacy Invasion and Data Misuse as Psychological Barriers to Peer-to-Peer Collaborative Car Use
}

Marcel Hunecke ${ }^{1}$, Nadine Richter ${ }^{1}$, and Holger Heppner ${ }^{1}$

${ }^{1}$ Faculty of Applied Social Sciences, University of Applied Sciences and Arts Dortmund

\section{Author Note}

We have no known conflict of interest to disclose.

Correspondence concerning this article should be addressed to Nadine Richter, University of Applied Sciences and Arts Dortmund, Emil-Figge-Straße 44, 44227 Dortmund, Germany, Email: nadine.richter@fh-dortmund.de

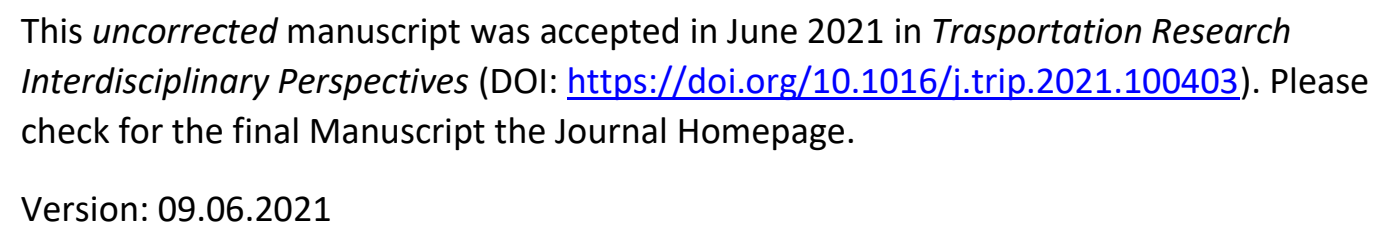




\begin{abstract}
The present study aimed to identify psychological barriers which potentially prevent individuals from implementing collaborative car use in their every-day mobility behaviour. We suggested a model consisting of four psychological barriers: Autonomy Loss, Privacy Invasion, Interpersonal Distrust, and Data Misuse. Perceived Financial Benefit was included as a main incentive for collaborative car use. Using two samples, a community $(N=176)$ and a student sample $(N=265)$, three forms of peer-to-peer collaborative car use were examined: lending your own car to another private person (Lending To), renting a car from another private person (Renting From) and sharing rides with others (Ridesharing). For all three forms, a standardised questionnaire was developed which included the psychological barriers, self-reported collaborative car use intention and behaviour, and evaluations of scenarios. The results showed that different barriers predicted specific forms of collaborative car use: Autonomy Loss was connected negatively with Ridesharing and Privacy Invasion predicted Lending To negatively. Data Misuse was related negatively with Renting From, when the renting was arranged via internet. Interpersonal Distrust showed no predictive value for collaborative car use. Perceived Financial Benefit was a consistent incentive for all forms of collaborative car use. Overall, the results confirm the relevance of psychological barriers for collaborative car use. Practical implications to overcome the psychological barriers are discussed.
\end{abstract}

Keywords: collaborative car use, carsharing, ridesharing, psychological barriers 


\section{Introduction}

Individual, private car use is the main mode of transport in highly industrialised nations (Urry, 2004). The transport sector as a whole is responsible for about $23 \%$ of global energy related greenhouse-gases (GHG) and other emissions such as soot and particulate matter (World Health Organization, 2018), that are related to a variety of global and local problems. Globally, the climate crisis calls for a reduction of GHG, locally, pollution caused by cars threatens health and quality of life, especially in bigger cities (Kumar et al., 2014; Stanley et al., 2018). An effective strategy to increase the occupancy rate of cars is achieved best by encouraging people to use cars collaboratively, for example through ridesharing (Furuhata et al., 2013). Privately organised peer-topeer carsharing, has a high potential to effectively increase the capacity occupancy rate of cars (Dill et al., 2019). Shifting to more environmentally friendly behaviour like collaborative car use is often inhibited by structural barriers that hinder the behaviour's implementation due to an increased effort. To decrease structural barriers, Business-to consumer (B2C) carsharing providers minimize organisational effort through an easy access to close by vehicles and information- and communication technologies (ICT) (Cohen-Blankshtain and Rotem-Mindali, 2016; Smith et al., 2020). Moreover, peer-to-peer (P2P) organised collaborative car use has become easier and more flexible through ICT. However, despite the elimination of structural barriers by using convenient technical solutions as well as the obvious financial benefits, collaborative car use is still not a very common practice in german-speaking countries (ifmo, 2016). Consequently, it is likely that additional psychological barriers prevent people from collaborative car use. Those psychological barriers refer to cognitive, attitudinal and social factors that prevent an individual from behavioural changes, even if they are convinced that it would be useful (Gifford, 2011). The present study therefore focuses on psychological barriers to peer-to-peer collaborative car use and investigates their role for behavioural intentions and behaviours. Thereby, the study contributes to a better understanding of the demand side for ICT-supported organization of collective mobility (Wang and Yang, 2019). 
Both B2C as well as P2P forms of collaborative car use have high potentials for increasing the occupation rate of cars. Nevertheless, P2P forms of collaborative car use are easier to implement, because business models and the maintenance of vehicle pools are not necessary. Therefore, the present study focuses on three forms of P2P collaborative car use that are easy to implement: lending a private car to others (Lending To), renting a car from others (Renting From) and travelling in a car with others (Ridesharing).

\section{Psychological Barriers to Peer-to-Peer Collaborative Car Use}

Psychological factors related to car use in general have been thoroughly examined in multiple empirical studies and meta-analyses (Gardner and Abraham, 2008; Hoffmann et al., 2017; Lanzini and Khan, 2017) and different empirical-based conceptual frameworks have been derived from their results (Chng et al., 2018; Pronello and Gaborieau, 2018). Most studies are theoretically based on Ajzen's (1991) Theory of Planned Behaviour (TPB) (Bamberg et al., 2003; Haustein and Hunecke, 2007; Gardner and Abraham, 2010) according to which the intention to use a car is formed by perceived behaviour control (PBC) , attitude and subjective norms. These factors are often extended by additional variables such as personal norms from Schwartz's (1977) Norm-Activation Model (NAM) (Heath and Gifford, 2002; Nordlund and Garvill, 2003; Bamberg et al., 2007) or habit (Verplanken et al., 1998; Ramos et al., 2020). Considering symbolic-affective dimensions of car use as a complementary perspective, studies showed that motives such as autonomy and privacy are related to specific travel mode choices and play an important role for evaluation processes of mobility intentions and behaviours (Steg, 2005; Hunecke et al., 2007; Lois and López-Sáez, 2009).

So far, only a few studies have considered psychological factors in relation to collaborative forms of car use. Studies on carpooling found that PBC is an important predictor for carpooling intention (Abrahamse et al., 2009; Bachmann et al., 2018). In another investigation, the psychological need for car ownership was found to be negatively related to collaborative car use intention 
(Paundra et al., 2017). Results of a study on subjective attitudes of carsharing moreover found that station-based carsharing users view a car mainly as a means to an end whereas users of free-floating carsharing associate a large symbolic and emotional value with a car (ifmo, 2016). Schaefers (2013) identified value-seeking, convenience, lifestyle, and environmental motives as four central motives for carsharing. Tussyadiah (2015) in turn identified societal aspects of sustainability and financial benefits as incentives and a lack of trust as well as unsatisfactory efficiency of technology as hindrances to engaging in mobility sharing concepts. In conclusion a systematic and comprehensive examination of potential psychological barriers discouraging individuals from collaborative car use is missing up to now. In view of the established psychological predictors of car use and the differences between private and collaborative car use, we suggest focusing on four psychological barriers to collaborative car use which are: Autonomy Loss, Privacy Invasion, Interpersonal Distrust, and Data Misuse:

Autonomy Loss refers to a perceived limited flexibility and control in collaborative car use compared to private car use. A qualitative study of commuters by Thomas et al. (2014) found concerns regarding Autonomy Loss to be a major reason against shifting their travel mode away from private car use. In the context of psychological action theories, beliefs of autonomy are conceptualized as self-efficacy, or, with regard to the TPB, as PBC. PBC has been consistently found to be one of the most important predictors for private car use (Gardner and Abraham, 2008). Haustein and Hunecke (2007) further showed that, additionally to PBC, a perceived necessity to be mobile at all times is related to private car use and decreases the desire for alternative modes of mobility. Indeed, compared to private car use, the collaborative use of cars is accompanied by conditions that can be perceived as a restriction of autonomy. For example, when using cars collaboratively, there is no permanent availability of a particular vehicle. Also, participants depend on the organisation and infrastructure offered by the commercial or peer provider. Before starting a journey, a car has to be found online, booked and physically localized. Even if no problems occur during this process, the organisational effort is higher compared to using a private car. 
Privacy is spatially provided through the protection of one's own personal space from intrusion by other people and self-determined privacy regulation is an important precondition for subjective well-being (Altman, 1975). Privacy Invasion results from perceived violations of personal space by others. In different public transport situations proximity to others has been shown to be a relevant factor that causes stress and consequently has a repelling effect (Evans and Wener, 2007; Pham et al., 2015). Similarly, during ridesharing participants share a limited space with strangers, possibly over a longer period of time. Feeling uncomfortable or even unsafe is an important issue during ridesharing, especially for women (Sarriera et al., 2017). Furthermore, owning a car is often understood as having a private room and extended personal space (Fraine et al., 2007). Consequently, there are suggestions to design autonomous vehicles as "extended living rooms" (Sanguinetti et al., 2018). Therefore, Privacy Invasion might be perceived when lending a private car to strangers or renting a car from another private person.

Interpersonal Distrust refers to a lack of trust in other participants or providers when lending a car, renting a car or participating in ridesharing. Depending on the form of collaborative car use, distrust can refer to worrying that others might not take good care of your car, will reliably provide a car on time and in a certain condition, or simply be a pleasant, nonthreatening passenger. In P2P collaborative car use, the direct communication with other private people is necessary and thus Interpersonal Distrust has an important function. Lack of trust was found to be a major reason for keeping participants from lending their car to strangers (Shaheen et al., 2012; Ballús-Armet et al., 2014). Bachmann et al. (2018) also found dispositional trust to have an indirect effect on carpooling intention through PBC.

Data Misuse refers to the perceived risk of violation of data privacy and general uncertainty as to whether private data is handled confidentially. Sharing some personal information and data is often unavoidable when participating in a B2C or P2P collaborative car use system (Groth, 2019; Zhang et al., 2020). If participants are unsure if their personal data will be protected reliably, they will 
be less likely to participate (Bossauer et al., 2020). Indeed, the availability of private details of others such as home or work address, can pose serious safety issues for participants.

We assume that the four suggested psychological barriers discourage people from Lending To, Renting From and Ridesharing behaviours. In addition, each of the suggested forms of collaborative car use offers Financial Benefits, which are the most obvious and biggest incentive to engage in collaborative car use (Luca and Di Pace, 2015). Renting From others and Ridesharing can save money in comparison to maintaining a private car and by lending your car to others for a fee, financial benefits are easily accessible. Therefore, we include financial benefits as an additional variable in the present study. Table 1 summarizes the theoretically retrieved perceived restrictions related to the psychological barriers as well as financial benefits as an incentive.

\section{Table 1}

Perceived restrictions of psychological barriers to peer-to-peer collaborative car use

\begin{tabular}{|c|c|c|c|c|c|}
\hline \multirow[b]{2}{*}{$\begin{array}{c}\text { Form of } \\
\text { Collaborative } \\
\text { Car Use }\end{array}$} & \multicolumn{4}{|c|}{ Psychological barriers } & Benefits \\
\hline & $\begin{array}{l}\text { Autonomy } \\
\text { Loss }\end{array}$ & $\begin{array}{l}\text { Privacy } \\
\text { Invasion }\end{array}$ & $\begin{array}{l}\text { Interpersonal } \\
\text { Distrust }\end{array}$ & Data Misuse & Financial Benefits \\
\hline Lending To & $\begin{array}{l}\text { organisatio- } \\
\text { nal effort }\end{array}$ & $\begin{array}{c}\text { violation of } \\
\text { own private } \\
\text { space } \\
\end{array}$ & $\begin{array}{c}\text { user } \\
\text { unknown }\end{array}$ & $\begin{array}{c}\text { private data at } \\
\text { risk }\end{array}$ & earning money \\
\hline Renting From & $\begin{array}{l}\text { reduced } \\
\text { mobility }\end{array}$ & $\begin{array}{l}\text { violation of } \\
\text { another's } \\
\text { private space }\end{array}$ & dependency & $\begin{array}{c}\text { private data at } \\
\text { risk }\end{array}$ & saving money \\
\hline Ridesharing & $\begin{array}{l}\text { organisatio- } \\
\text { nal effort }\end{array}$ & $\begin{array}{c}\text { interaction } \\
\text { with } \\
\text { strangers }\end{array}$ & $\begin{array}{c}\text { feeling } \\
\text { uncomfortable } \\
\text { or unsafe }\end{array}$ & $\begin{array}{c}\text { private data at } \\
\text { risk }\end{array}$ & saving money \\
\hline
\end{tabular}

In an empircal study, we designed and empirically tested a standardized questionnaire that measures the proposed psychological barriers and financial benefits regarding the three forms of collaborative mobility [Q1]. Secondly, we examined if the proposed psychological barriers predict collaborative mobility intentions and behaviours [Q2]. 


\section{Method}

\subsection{Participants}

We collected data from two samples in two cities in the Ruhr area in Germany. The first was a community sample from the City of Bottrop (population of 117.000) and the second sample consisted of undergraduate students studying in Dortmund (population of 585.000).

\subsubsection{Sample 1: Community Sample}

We collected data in two different districts of the City of Bottrop (Stadtwald and Welheim) with a comparable infrastructure, such as a similar distance to the city centre, the railway station, and road infrastructure. Recruitment of participants was carried out in four phases. First, a letter was sent to all households with an invitation to participate in a study about "the shared use of cars" via an online survey or a personal interview. As an incentive, a five-euro shopping voucher was offered. The interviews took about 30 to 40 minutes, while the completion of the online version took about 20 minutes. Second, trained interviewers visited houses and asked the residents if they would like to participate. Interested households were either asked if they would like to arrange a personal standardized interview, were given a paper-pencil version of the survey or were referred to the online version of the survey. Third, a reminder including an invitation to participate was sent via post to households that had not yet agreed to participate. Fourth, interviewers recruited people in public spaces (e.g. in front of a shopping centre) if they would like to fill out the paper-pencil questionnaire. We thus contacted each household at least once via post.

Surveys from 206 participants were collected, with 30 participants being excluded because they came from a different place of residence than the targeted area or because they did not pass one of the two quality check items in the online survey. Thus, 176 cases remained for analysis. In total, a final participation rate of $2.5 \%$ was reached based on 6.921 residents above the age of 18 living in the contacted households. The mean age of the participants was 53.3 years (SD=14.5), $54 \%$ were male, $45.5 \%$ female and $0.6 \%$ indicated another gender. The majority were working as 
employees (54\%) or were retired (27.9\%). Most of the participants lived in two-person (50.6\%) or three-person households (18.1\%) with no children under the age of $18(75 \%)$. The majority possessed one (38.1\%) or two cars $(57.7 \%)$ and only four participants $(2.3 \%)$ stated that their household did not own a car. Detailed sample characteristics can be found in Appendix A.

\subsubsection{Sample 2: Student Sample}

To ensure that only undergraduate students participated, the study invitation was sent via an internal distribution list of the Dortmund University of Applied Sciences and Arts which reaches about 14.000 registered students. Additionally, a paper-pencil version of the survey was distributed in courses and the invitation was publicised in local online student communities. Due to a high response rate and to ensure a sample size comparable to the community sample, the survey was closed after only a few days.

We collected 320 finished online surveys and 22 paper-pencil surveys from undergraduate students. After removing 77 cases that did not pass one of the two quality check items in the online survey correctly, 265 participants remained for the analyses. The mean age was 24.2 years $(S D=4.4)$, $44.5 \%$ were male, $54.7 \%$ female and $0.8 \%$ other. $92.5 \%$ of the participants declared their main occupation was studying at a university. The majority were living in two-person (32.1\%) or threeperson households (24.5\%) with no children (86.4\%). $41.9 \%$ owned one car, $32.1 \%$ had two cars in their household and $23.8 \%$ stated no car ownership. About half (55.1\%) of the participants stated that they studied or worked in the same city as they lived. Additional details regarding the sample can be found in Appendix A. 


\subsection{Measures}

\subsubsection{Psychological Barriers and Perceived Financial Benefits}

In order to measure the potential psychological barriers and the main incentive Perceived Financial Benefits, theory-driven items were formulated for each form of P2P collaborative car use. A statistical pre-test of the items was conducted before the start of the main study with 72 participants. Distributions and factorial structure of the items and scales were analysed using exploratory factor analysis. Based on the results of the pretest some items were re-worded or removed. Table 2 shows examples of the final items for each barrier and behaviour that were used in the present study. A full list of the items is attached in Appendix B.

\section{Table 2}

Sample items for each barrier/benefit and form of P2P collaborative car use

\begin{tabular}{|c|c|c|c|}
\hline $\begin{array}{c}\text { Barrier / } \\
\text { Benefit }\end{array}$ & Lending To & Renting From & Ridesharing \\
\hline $\begin{array}{l}\text { Autonomy } \\
\text { Loss }\end{array}$ & $\begin{array}{l}\text { I find it organisationally } \\
\text { complex to offer my } \\
\text { car for rental. }\end{array}$ & $\begin{array}{l}\text { I consider the arrangements } \\
\text { for organising the rental of } \\
\text { a car from another private } \\
\text { person an effort. }\end{array}$ & $\begin{array}{l}\text { I do not like handing } \\
\text { over control of a } \\
\text { car to someone } \\
\text { else. }\end{array}$ \\
\hline $\begin{array}{l}\text { Privacy } \\
\text { Invasion }\end{array}$ & $\begin{array}{l}\text { My car is my private } \\
\text { space which I do not } \\
\text { want to share with } \\
\text { strangers. }\end{array}$ & $\begin{array}{l}\text { It is unpleasant for me to use } \\
\text { a car of an unknown } \\
\text { person, because doing this } \\
\text { invades his/her privacy. }\end{array}$ & $\begin{array}{l}\text { To sit in a car with a } \\
\text { stranger is } \\
\text { unpleasant for me. }\end{array}$ \\
\hline $\begin{array}{l}\text { Interpersonal } \\
\text { Distrust }\end{array}$ & $\begin{array}{l}\text { When I rent my car, I } \\
\text { worry that it will be } \\
\text { damaged. }\end{array}$ & $\begin{array}{l}\text { If I rented a car from a private } \\
\text { person, I would be } \\
\text { concerned that he or she } \\
\text { might be unreliable. }\end{array}$ & $\begin{array}{l}\text { I feel unsafe sitting in } \\
\text { a car while } \\
\text { Ridesharing with } \\
\text { strangers. }\end{array}$ \\
\hline Data Misuse & $\begin{array}{l}\text { I have no trust in the } \\
\text { privacy policy of } \\
\text { internet platforms that } \\
\text { organise the rental of } \\
\text { private cars. }\end{array}$ & $\begin{array}{l}\text { I have no trust in the data } \\
\text { privacy of internet } \\
\text { platforms where I can rent } \\
\text { a car from a private } \\
\text { person. }\end{array}$ & $\begin{array}{l}\text { I have no trust in the } \\
\text { data privacy of } \\
\text { internet platforms } \\
\text { that organise } \\
\text { Ridesharing. }\end{array}$ \\
\hline $\begin{array}{l}\text { Perceived } \\
\text { Financial } \\
\text { Benefits }\end{array}$ & $\begin{array}{l}\text { By renting out my car I } \\
\text { would have financial } \\
\text { advantages. }\end{array}$ & $\begin{array}{l}\text { I can save money by renting a } \\
\text { car from a private person } \\
\text { for my everyday trips. }\end{array}$ & $\begin{array}{l}\text { I can save money } \\
\text { through } \\
\text { Ridesharing. }\end{array}$ \\
\hline
\end{tabular}




\subsubsection{Variables from the Theory of Planned Behaviour and Personal Norms}

To test nomological validity of the psychological barriers and perceived Financial Benefits, central variables of theories of action (TPB, NAM) were assessed. Based on the recommendations of Ajzen (2006) items for social norms, intention, PBC and attitude were formulated regarding collaborative mobility behaviour. Additionally, personal norms as a central variable of the normactivation model were included (Schwartz, 1977). A full list of the items is attached in Appendix B.

\subsubsection{Collaborative Car Use, Intentions and Scenarios}

Self-reported frequency of P2P collaborative car use behaviour was assessed by one item each using a 5-point Likert scale ranging from 1 "never" to 5 "always". As a very low use of collaborative car use in general was assumed and in order to reach a greater variance, additional items, that describe scenarios of P2P collaborative car use were developed. The aim was to present scenarios that represent realistic and most likely P2P collaborative car use behaviours. For each of the three behaviours, two scenarios were developed. One focused on engaging in collaborative mobility via a personal contact with neighbours, and the other focused on contact via an internet platform. Participants were asked to rate their willingness to participate in scenario situations ("Would you use this offer?") on a 5-point scale ranging from 1 "certainly not" to 5 "for certain". A full list of all scenarios is attached in Appendix B.

\subsection{Data Analysis}

The factorial structure of the psychological barriers/benefits was tested with confirmatory factor analysis (CFA) using lavaan (Rosseel, 2012). The model fit for the CFA was assessed using the Comparative Fit Index (CFI), Tucker-Lewis Index (TLI), Root Mean Square Error of Approximation (RMSEA), and the Standardized Root Mean Square Residual (SRMR). CFI and TLI of $\geq .95$, an RMSEA, and an SRMR of $\leq .08$ indicate an acceptable fit (Hu and Bentler, 1999). As the data was not multivariately normally distributed, the Satorra-Bentler correction for the test statistics was applied. 
In some cases, theoretically similar items were summarized as parcels to maintain a statistical power as high as possible. Based on the CFA, scales were summarized for further analyses and Cronbach'salpha as well as bivariate correlations were calculated for each sample and form of collaborative car use.

In order to analyse relationships between the proposed barriers/benefits and intentions as well as the self-reported frequency of behaviour, regression analysis was performed. Because the intention and behaviour variables had skewed distributions, they were dichotomised. Participants who reported that they never used or intended to use any of the suggested forms of P2P collaborative car use were separated from participants who reported that they perform or intend to perform those behaviours at least occasionally. The dichotomized variables were used in a logistic regression as dependent variables. Nagelkerkes pseudo- $\mathrm{R}^{2}$ was calculated as indicator of model quality. Values of $>.20$ are considered acceptable, $>.40$ good and $>.50$ very good. Additionally, the degree of motorization was calculated (number of cars/adults in a household) and included as a control variable in the models with Lending To and Renting From. The relationships between the proposed barriers/benefits and the different scenarios were analysed using path models. In these models, barriers and benefits were allowed to covariate. In general, cases were excluded listwise from the analyses, which led to different sample sizes. In all analyses with Renting To as a dependent variable, only participants with a driving license and at least one car in their household were included. 


\section{Results}

\subsection{Confirmatory Factor Analyses, Internal Consistencies and Intercorrelations of the Final Scales}

In the community sample, the CFA yielded an overall acceptable fit (see Table 3). Also in the student sample, the models showed acceptable fit indices for the three forms of P2P collaborative car use (see Table 4). The item loadings and intercorrelations of the different factors are summarised in Figures 1-3.

Table 3

Model fit indices of the CFA (community sample)

\begin{tabular}{lcccccc}
\hline & \multicolumn{1}{c}{$\mathbf{X}^{2}$-test } & RMSEA $[90 \%$ CI] & SRMR & CFI & TLI & $\boldsymbol{N}$ \\
\hline Lending To & $\mathrm{X}^{2}(12)=15.96, p=.193$ & $.058[.000, .126]$ & .037 & .99 & .96 & 162 \\
Renting From & $\mathrm{X}^{2}(18)=36.81, \mathrm{p}=.006$ & $.089[.047, .130]$ & .048 & .95 & .90 & 167 \\
Ridesharing & $\mathrm{X}^{2}(18)=44.07, \mathrm{p}=.001$ & $.106[.066, .146]$ & .049 & .95 & .88 & 168 \\
\hline
\end{tabular}

Note. Sattora-Bentler-Correction was applied. Participants without a car were removed in the Lending To and Renting From-models.

\section{Table 4}

Model fit indices of the CFA (student sample)

\begin{tabular}{llccccc}
\hline & $\mathbf{X}^{2}$-test & RMSEA $[90 \%$ CI $]$ & SRMR & CFI & TLI & $\boldsymbol{N}$ \\
\hline Lending To & $\mathrm{X}^{2}(12)=12.92, \mathrm{p}=.375$ & $.023[.000, .088]$ & .028 & .99 & .99 & 194 \\
Renting From & $\mathrm{X}^{2}(18)=29.23, \mathrm{p}=.046$ & $.053[.008, .088]$ & .035 & .98 & .96 & 262 \\
Ridesharing & $\mathrm{X}^{2}(18)=33.36, \mathrm{p}=.015$ & $.060[.026, .092]$ & .030 & .98 & .97 & 261 \\
\hline
\end{tabular}

Note. Sattora-Bentler-Correction was applied. Participants without a car were removed in the Renting to-models. 


\section{Figure 1}

\section{CFA "Lending To"}

Community Sample

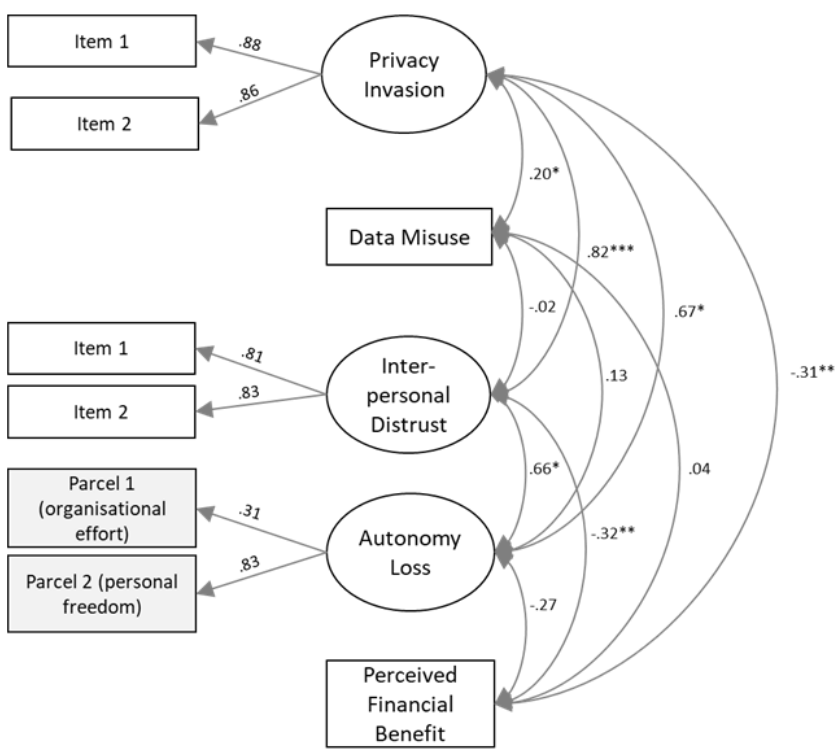

Student Sample

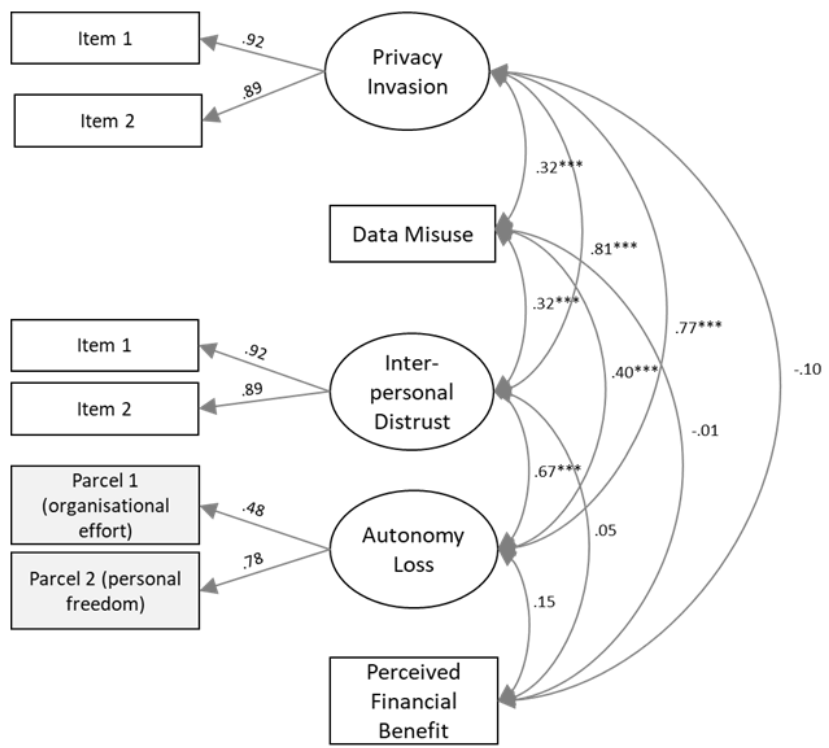

Note. ${ }^{*} \mathrm{~b}<.05 ;{ }^{* *} \mathrm{~b}<.01 ;{ }^{* * *} \mathrm{~b}<.001 ;$ standardized coefficients.

\section{Figure 2}

\section{CFA "Renting From"}

Community Sample

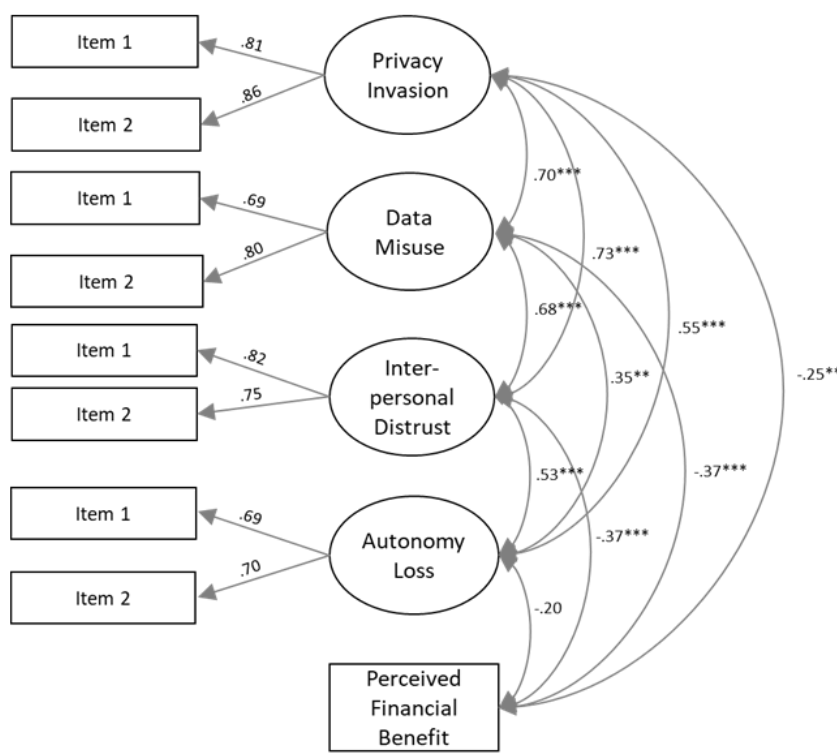

Student Sample

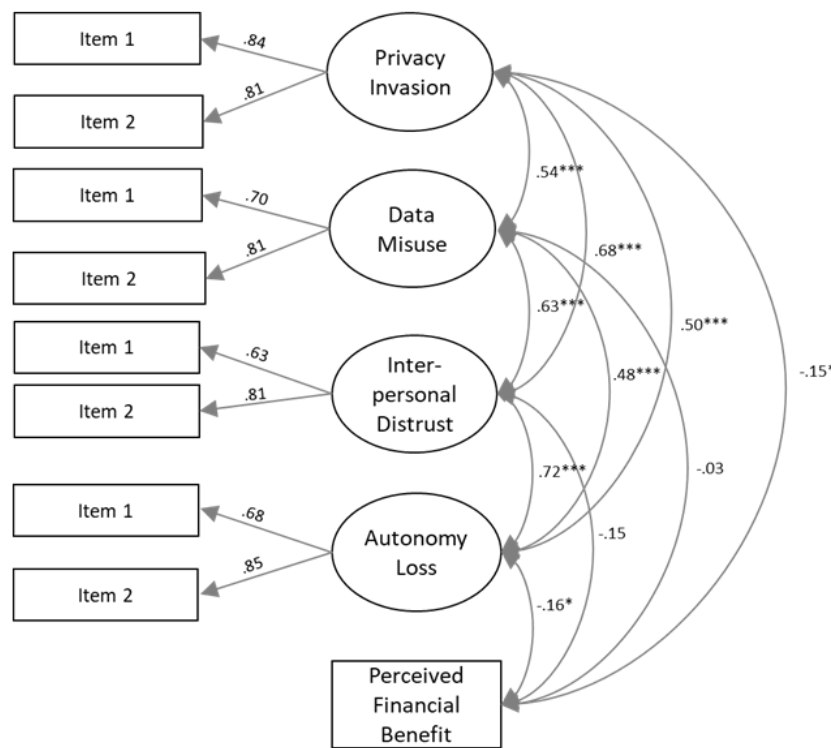

Note. ${ }^{*} \mathrm{~b}<.05 ;{ }^{*} \mathrm{~b}<.01 ;{ }^{* * *} \mathrm{~b}<.001 ;$ standardized coefficients. 


\section{Figure 3}

\section{CFA Ridesharing}

Community Sample

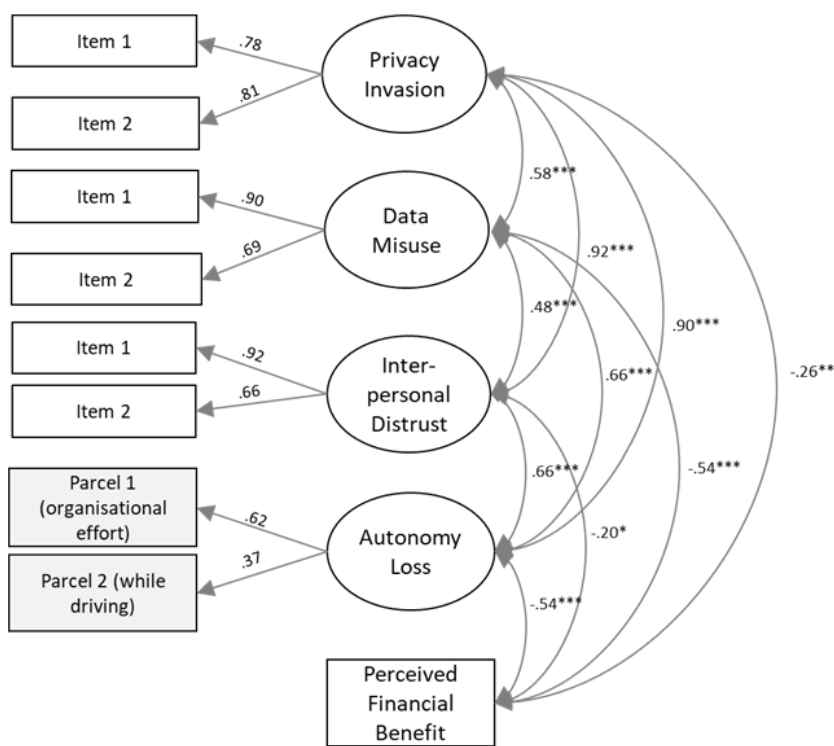

Student Sample

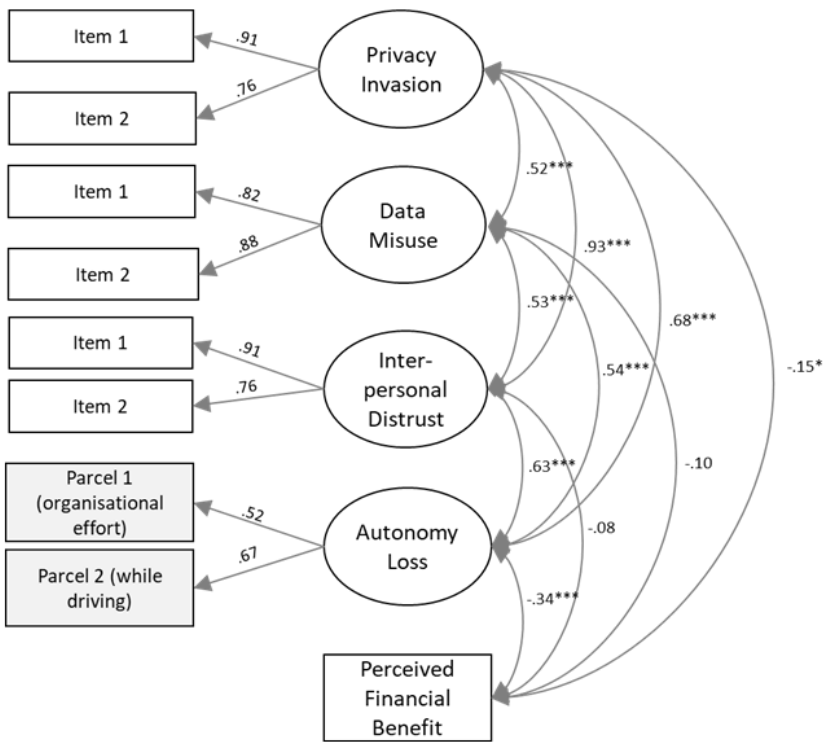

Note. ${ }^{*} \mathrm{~b}<.05 ;{ }^{*} \mathrm{~b}<.01 ;{ }^{* * *} \mathrm{~b}<.001 ;$ standardized coefficients.

Based on the described analyses, we aggregated the final scales Autonomy Loss, Privacy Invasion, Interpersonal Distrust, Data Misuse, and perceived Financial Benefit for further analysis. Overall, most of these scales showed acceptable to very good internal consistencies ranging from $\alpha=$ .67 to .90 . Only the Autonomy Loss scales with regard to Renting To and Ridesharing showed a low internal consistency in the community sample $(\alpha=.60)$. The scales with regard to collaborative car use in general (goal intention, attitude, perceived behaviour control, social norms, personal norms) showed good internal consistencies from $\alpha=.72$ to .93 with exception of the perceived behaviour control scale in the community sample, which was considerably lower $(\alpha=.62)$. The intercorrelations of the barriers with the other general scales were medium-sized and in the expected direction, which is an indicator for nomological validity of the suggested psychological barriers. Descriptive sample characteristics, internal consistencies of the scales and their intercorrelations for each sample and form of collaborative car use are summarized in Appendix C. 


\subsection{Logistic Regressions and Path Models}

\subsubsection{Lending To}

4.2.1.1 Community Sample. The logistic regressions of Lending To with intention as a dependent variable revealed the barrier Privacy Invasion as a significant predictor $(\mathrm{OR}=.44, \mathrm{p}=$ .001). Perceived Financial Benefit showed a positive relationship with intention $(\mathrm{OR}=1.46, \mathrm{p}=.048)$.

Further, Privacy Invasion appeared to be a significant predictor (OR $=.56, p=.012$ ) for behaviour.

The path models with the barriers as predictors for the scenario ratings showed a similar pattern as the logistic regressions. Privacy Invasion appeared to be a medium strong significant predictor for both the neighbour-scenario $(\beta=-.59, p<.001)$ and the internet-scenario ratings $(\beta=-.41, p<.001)$. Besides the barrier Privacy Invasion, the perceived Financial Benefit showed a small positive relationship with the evaluation of the neighbour-scenario $(\beta=.13, p=.036)$ as well as a medium strong relationship with the internet-scenario $(\beta=.34, p<.001)$. The analyses are summarised in Table 5.

\section{Table 5}

"Lending To": Odds ratios on intention and behaviour and path coefficients on scenario ratings (community sample)

\begin{tabular}{|c|c|c|c|c|c|c|c|c|c|c|}
\hline & \multicolumn{6}{|c|}{ Logistic Regressions } & \multicolumn{4}{|c|}{ Path analysis } \\
\hline & \multicolumn{3}{|c|}{$\begin{array}{l}\text { Intention } \\
(\mathrm{N}=156)\end{array}$} & \multicolumn{3}{|c|}{$\begin{array}{l}\text { Behaviour } \\
(\mathrm{N}=155)\end{array}$} & \multicolumn{2}{|c|}{$\begin{array}{l}\text { Neighbour- } \\
\text { Scenario } \\
(\mathrm{N}=168)\end{array}$} & \multicolumn{2}{|c|}{$\begin{array}{l}\text { Internet- } \\
\text { Scenario } \\
(\mathrm{N}=165)\end{array}$} \\
\hline & OR & $p$ & $\mathrm{Cl}$ & OR & $p$ & $\mathrm{Cl}$ & $\beta$ & $\mathrm{p}$ & $\beta$ & $p$ \\
\hline $\begin{array}{l}\text { Autonomy } \\
\text { Loss }\end{array}$ & 1.03 & .928 & $\begin{array}{l}.588 \\
1.79\end{array}$ & .85 & .804 & $\begin{array}{l}.536 \\
1.35\end{array}$ & -.14 & .063 & -.11 & .166 \\
\hline $\begin{array}{l}\text { Privacy } \\
\text { Invasion }\end{array}$ & .44 & .001 & $\begin{array}{l}.266 \\
.718\end{array}$ & .56 & .012 & $\begin{array}{l}.365 \\
.880\end{array}$ & -.59 & $<.001$ & -.41 & $<.001$ \\
\hline $\begin{array}{l}\text { Interpersonal } \\
\text { Distrust }\end{array}$ & .89 & .691 & $\begin{array}{l}.509 \\
1.56\end{array}$ & 1.02 & .804 & $\begin{array}{l}.648 \\
1.75\end{array}$ & .11 & .213 & .12 & .234 \\
\hline Data Misuse & .95 & .758 & $\begin{array}{l}.689 \\
1.31\end{array}$ & 1.13 & .371 & $\begin{array}{l}.864 \\
1.49\end{array}$ & - & - & -.04 & .559 \\
\hline $\begin{array}{c}\text { Perceived } \\
\text { Financial } \\
\text { Benefit }\end{array}$ & 1.46 & .048 & $\begin{array}{l}1.00 \\
2.13\end{array}$ & 1.02 & .902 & $\begin{array}{l}.731 \\
1.43\end{array}$ & .13 & .036 & .34 & $<.001$ \\
\hline Pseudo- $\mathrm{R}^{2}$ & .34 & & & .15 & & & & & & \\
\hline $\begin{array}{c}\text { correctly } \\
\text { predicted (\%) }\end{array}$ & 79.5 & & & 69.0 & & & & & & \\
\hline $\mathrm{R}^{2}$ & & & & & & & .38 & & .31 & \\
\hline
\end{tabular}


Note. The logistic regression models are controlled for degree of motorization (number of cars/adults in household). List-wise exclusion of cases was used, $\mathrm{OR}=$ Odds Ratio, $\mathrm{Cl}=90 \%$ Confidence Interval, Pseudo- $R^{2}=$ Nagelkerkes $R^{2}$

4.2.1.2 Student Sample. The logistic regression yielded a similar pattern as in the community sample (see Table 6). Privacy Invasion ( $O R=.51, p=.001)$ as well as Data Misuse $(\mathrm{OR}=.71, \mathrm{p}=.024)$ are significant predictors that decreased the intention to lend your car to another private person. Also, for the path models, Privacy Invasion was a significant predictor for the neighbour-scenario rating ( $\beta=-.20, p=.048)$ as well as for the internet-scenario rating $(\beta=-.36, p<.001)$. Furthermore, as in the student sample, perceived Financial Benefit showed positive small to medium-sized weights for both scenario ratings (neighbour: $\beta=.25, p<.001$; internet: $\beta=.14, p=.018$ ).

\section{Table 6}

"Lending To": Odds ratios on intention and behaviour and path coefficients on scenario ratings (student sample)

\begin{tabular}{|c|c|c|c|c|c|c|c|c|c|c|}
\hline & \multicolumn{6}{|c|}{ Logistic Regressions } & \multicolumn{4}{|c|}{ Path Models } \\
\hline & \multicolumn{3}{|c|}{$\begin{array}{l}\text { Intention } \\
(N=193)\end{array}$} & \multicolumn{3}{|c|}{$\begin{array}{l}\text { Behaviour } \\
(N=193)\end{array}$} & \multicolumn{2}{|c|}{$\begin{array}{l}\text { Neighbour- } \\
\text { Scenario } \\
(N=195)\end{array}$} & \multicolumn{2}{|c|}{$\begin{array}{c}\text { Internet- } \\
\text { Scenario } \\
(\mathrm{N}=195)\end{array}$} \\
\hline & OR & $\mathbf{p}$ & $\mathrm{Cl}$ & OR & $\mathbf{p}$ & $\mathrm{Cl}$ & $\beta$ & $p$ & $\beta$ & $p$ \\
\hline Autonomy Loss & .88 & .540 & $\begin{array}{l}.585, \\
1.33\end{array}$ & 1.14 & .479 & $\begin{array}{l}.792, \\
1.64\end{array}$ & -.15 & .064 & -.05 & .544 \\
\hline $\begin{array}{l}\text { Privacy } \\
\text { Invasion }\end{array}$ & .51 & .001 & $\begin{array}{l}.341 \\
.755\end{array}$ & .80 & .220 & $\begin{array}{l}.562, \\
1.10\end{array}$ & -.20 & .048 & -.36 & $<.001$ \\
\hline $\begin{array}{l}\text { Interpersonal } \\
\text { Distrust }\end{array}$ & .74 & .736 & $\begin{array}{l}.583, \\
1.46\end{array}$ & .89 & .533 & $\begin{array}{l}.580, \\
1.33\end{array}$ & -.13 & .156 & -.16 & .076 \\
\hline Data Misuse & .71 & .024 & $\begin{array}{l}.531, \\
.957\end{array}$ & 1.10 & .453 & $\begin{array}{l}.853, \\
1.43\end{array}$ & - & - & -.07 & .296 \\
\hline $\begin{array}{c}\text { Perceived } \\
\text { Financial } \\
\text { Benefit }\end{array}$ & 1.22 & .180 & $\begin{array}{l}.912 \\
1.64\end{array}$ & .93 & .618 & $\begin{array}{l}.721 \\
1.21\end{array}$ & .25 & $<.001$ & .14 & .018 \\
\hline Pseudo- $R^{2}$ & .30 & & & .04 & & & & & & \\
\hline $\begin{array}{c}\text { correctly } \\
\text { predicted (\%) }\end{array}$ & 70.0 & & & 55.4 & & & & & & \\
\hline $\mathrm{R}^{2}$ & & & & & & & .23 & & .32 & \\
\hline
\end{tabular}

Note. The logistic regression models are controlled for degree of motorization (number of cars/adults in household). List-wise exclusion of cases was used, $\mathrm{OR}=$ Odds Ratio, $\mathrm{Cl}=90 \%$ Confidence Interval, Pseudo- $\mathrm{R}^{2}=$ Nagelkerkes $\mathrm{R}^{2}$ 


\subsubsection{Renting From}

4.2.2.1 Community Sample. The logistic regression to explain the intention to rent cars from private people, revealed Perceived Financial Benefit as a significant predictor for the intention to rent a $\operatorname{car}(\mathrm{OR}=1.47, \mathrm{p}=.023)$. Further, Autonomy Loss was found to be a medium strong $(\beta=-.28, \mathrm{p}<$ .001) negative predictor for the neighbour-scenario ratings. In contrast, Perceived Financial Benefit was positively predicting the neighbour-scenario $(\beta=-.24, p=.001)$. In the case of the internetscenario, Privacy Invasion $(\beta=-.22, p=.013)$, Data Misuse $(\beta=-.23, p=.003)$ and Financial Benefits $(\beta=.18, p=.008)$ turned out to be significant predictors. The results are summarized in Table 7.

\section{Table 7}

"Renting From": Odds ratios on intention and behaviour and path coefficients on scenario ratings (community sample)

\begin{tabular}{|c|c|c|c|c|c|c|c|c|c|c|}
\hline & \multicolumn{6}{|c|}{ Logistic Regressions } & \multicolumn{4}{|c|}{ Path Models } \\
\hline & \multicolumn{3}{|c|}{$\begin{array}{l}\text { Intention } \\
(\mathrm{N}=165)\end{array}$} & \multicolumn{3}{|c|}{$\begin{array}{l}\text { Behaviour } \\
(\mathrm{N}=164)\end{array}$} & \multicolumn{2}{|c|}{$\begin{array}{c}\text { Neighbour- } \\
\text { Scenario } \\
(\mathrm{N}=172)\end{array}$} & \multicolumn{2}{|c|}{$\begin{array}{l}\text { Internet- } \\
\text { Scenario } \\
(N=171)\end{array}$} \\
\hline & OR & $p$ & $\mathrm{Cl}$ & OR & $\mathbf{p}$ & $\mathrm{Cl}$ & $\beta$ & $\mathbf{p}$ & $\beta$ & $\mathbf{p}$ \\
\hline Autonomy Loss & .68 & .089 & $\begin{array}{l}.434 \\
1.06\end{array}$ & .73 & .401 & $\begin{array}{l}.845, \\
1.52\end{array}$ & -.28 & $<.001$ & -.12 & .104 \\
\hline $\begin{array}{l}\text { Privacy } \\
\text { Invasion }\end{array}$ & .75 & .209 & $\begin{array}{l}.485 \\
1.17\end{array}$ & 1.05 & .819 & $\begin{array}{l}.714, \\
1.53\end{array}$ & -.10 & .303 & -.22 & .013 \\
\hline $\begin{array}{l}\text { Interpersonal } \\
\text { Distrust }\end{array}$ & 1.23 & .395 & $\begin{array}{l}.765 \\
1.97\end{array}$ & .92 & .681 & $\begin{array}{l}.609 \\
1.38\end{array}$ & -.06 & .501 & .01 & .885 \\
\hline Data Misuse & .88 & .503 & $\begin{array}{l}.593, \\
1.29\end{array}$ & .83 & .303 & $\begin{array}{l}.585, \\
1.18\end{array}$ & - & - & -.23 & .003 \\
\hline $\begin{array}{c}\text { Perceived } \\
\text { Financial } \\
\text { Benefit }\end{array}$ & 1.47 & .023 & $\begin{array}{l}1.06 \\
2.04\end{array}$ & .92 & .880 & $\begin{array}{l}.306 \\
2.76\end{array}$ & .24 & .001 & .18 & .008 \\
\hline Pseudo- $\mathrm{R}^{2}$ & .15 & & & .07 & & & & & & \\
\hline $\begin{array}{c}\text { correctly } \\
\text { predicted (\%) }\end{array}$ & 78.8 & & & 67.7 & & & & & & \\
\hline $\mathrm{R}^{2}$ & & & & & & & .23 & & .27 & \\
\hline
\end{tabular}

Note. The logistic regression models are controlled for degree of motorization (number of cars/adults in household). List-wise exclusion of cases was used, OR= Odds Ratio, $\mathrm{Cl}=90 \%$ Confidence Interval, Pseudo- $R^{2}=$ Nagelkerkes $R^{2}$

4.2.2.2 Student Sample. The analyses in the student sample for the rental of cars from other private people, shows that Perceived Financial Benefit is a highly significant predictor for 
Intention $(\mathrm{OR}=1.8, \mathrm{p}<.001)$. With regard to behaviour, the barrier Interpersonal Distrust was a significant predictor $(O R=.71, p=.036)$. As predictors of the neighbour-scenario, perceived Financial Benefit showed a significant relationship $(\beta=.20, p=.001)$. For the internet-scenario, as in the community sample, the barriers Privacy Invasion $(\beta=-.14, p=.033)$, Data Misuse $(\beta=-.26, p<.001)$ as well as Perceived Financial Benefit $(\beta=.32, p<.001$ ) were significant predictors (see Table 8).

\section{Table 8}

"Renting From": Odds ratios on intention and behaviour and path coefficients on scenario ratings (student sample)

\begin{tabular}{|c|c|c|c|c|c|c|c|c|c|c|}
\hline & \multicolumn{6}{|c|}{ Logistic Regressions } & \multicolumn{4}{|c|}{ Path Models } \\
\hline & \multicolumn{3}{|c|}{$\begin{array}{l}\text { Intention } \\
(\mathrm{N}=265)\end{array}$} & \multicolumn{3}{|c|}{$\begin{array}{c}\text { Behaviour } \\
(\mathrm{N}=258)\end{array}$} & \multicolumn{2}{|c|}{$\begin{array}{l}\text { Neighbour- } \\
\text { Scenario } \\
(\mathrm{N}=265)\end{array}$} & \multicolumn{2}{|c|}{$\begin{array}{l}\text { Internet- } \\
\text { Scenario } \\
(\mathrm{N}=265)\end{array}$} \\
\hline & OR & $p$ & $\mathrm{Cl}$ & OR & $\mathbf{p}$ & $\mathrm{Cl}$ & $\beta$ & $\mathbf{p}$ & $\beta$ & $\mathbf{p}$ \\
\hline $\begin{array}{l}\text { Autonomy } \\
\text { Loss }\end{array}$ & .77 & .116 & $\begin{array}{l}.553 \\
1.07\end{array}$ & .90 & .501 & $\begin{array}{l}.660 \\
1.23\end{array}$ & -.06 & .388 & -.06 & .317 \\
\hline $\begin{array}{l}\text { Privacy } \\
\text { Invasion }\end{array}$ & .99 & .950 & $\begin{array}{l}.751 \\
1.31\end{array}$ & 1.01 & .927 & $\begin{array}{l}.782, \\
1.31\end{array}$ & -.11 & .130 & -.14 & .033 \\
\hline $\begin{array}{l}\text { Interpersonal } \\
\text { Distrust }\end{array}$ & .91 & .558 & $\begin{array}{l}.652 \\
1.26\end{array}$ & .71 & .036 & $\begin{array}{l}.515 \\
977\end{array}$ & -.09 & .236 & -.03 & .664 \\
\hline Data Misuse & .84 & .195 & $\begin{array}{l}.638, \\
1.10\end{array}$ & 1.14 & .318 & $\begin{array}{l}.882 \\
1.47\end{array}$ & - & - & -.26 & $<.001$ \\
\hline $\begin{array}{c}\text { Perceived } \\
\text { Financial } \\
\text { Benefit }\end{array}$ & 1.80 & $<.001$ & $\begin{array}{l}1.43 \\
2.34\end{array}$ & 1.09 & .418 & $\begin{array}{l}.886 \\
1.34\end{array}$ & .20 & .001 & .32 & $<.001$ \\
\hline Pseudo- $R^{2}$ & .07 & & & .07 & & & & & & \\
\hline $\begin{array}{c}\text { correctly } \\
\text { predicted (\%) }\end{array}$ & 67.7 & & & 60.1 & & & & & & \\
\hline $\mathrm{R}^{2}$ & & & & & & & .10 & & .28 & \\
\hline
\end{tabular}

Note. The logistic regression models are controlled for degree of motorization (number of cars/adults in household). List-wise exclusion of cases was used, $\mathrm{OR}=$ Odds Ratio, $\mathrm{Cl}=90 \%$ Confidence Interval, Pseudo- $R^{2}=$ Nagelkerkes $R^{2}$ 


\subsubsection{Ridesharing}

4.2.3.1 Community Sample.The logistic regression with intention to use Ridesharing in every-day mobility as a dependent variable revealed Perceived Financial Benefit of Ridesharing as a strong positive predictor $(O R=1.79 ; p<.001)$. In contrast, only Autonomy Loss was significant in the model with Ridesharing behaviour as a dependent variable $(O R=.45, p=.001)$. For the neighbourscenario, Financial Benefit was a medium strong predictor $(\beta=.35, p<.001)$ as well as Autonomy Loss $(\beta=-.24, p=.002)$. For the internet-scenario, Perceived Financial Benefit showed a mediumstrong positive relationship $(\beta=.35, p<.001)$ and Data Misuse a medium strong to small predictor ( $\beta$ $=-.22, \mathrm{p}=.003)$. The results are summarized in Table 9.

\section{Table 9}

"Ridesharing": Odds ratios on intention and behaviour and path coefficients on scenario ratings (community sample)

\begin{tabular}{|c|c|c|c|c|c|c|c|c|c|c|}
\hline & \multicolumn{6}{|c|}{ Logistic Regressions } & \multicolumn{4}{|c|}{ Path Models } \\
\hline & \multicolumn{3}{|c|}{$\begin{array}{l}\text { Intention } \\
(N=171)\end{array}$} & \multicolumn{3}{|c|}{$\begin{array}{l}\text { Behaviour } \\
(N=162)\end{array}$} & \multicolumn{2}{|c|}{$\begin{array}{l}\text { Neighbour- } \\
\text { Scenario } \\
(N=173)\end{array}$} & \multicolumn{2}{|c|}{$\begin{array}{l}\text { Internet- } \\
\text { Scenario } \\
(\mathrm{N}=172)\end{array}$} \\
\hline & OR & $\mathbf{p}$ & $\mathrm{Cl}$ & OR & $\mathbf{p}$ & $\mathrm{Cl}$ & $\boldsymbol{\beta}$ & $p$ & $\beta$ & $\mathbf{p}$ \\
\hline $\begin{array}{l}\text { Autonomy } \\
\text { Loss }\end{array}$ & .66 & .109 & $\begin{array}{l}.399, \\
1.10\end{array}$ & .45 & .001 & $\begin{array}{l}.274 \\
.735\end{array}$ & -.24 & .002 & -.09 & .291 \\
\hline $\begin{array}{l}\text { Privacy } \\
\text { Invasion }\end{array}$ & .68 & .137 & $\begin{array}{l}.412 \\
1.13\end{array}$ & 1.11 & .648 & $\begin{array}{l}.703 \\
1.76\end{array}$ & -.09 & .380 & .08 & .429 \\
\hline $\begin{array}{l}\text { Interpersonal } \\
\text { Distrust }\end{array}$ & 1.50 & .080 & $\begin{array}{l}.952 \\
2.37\end{array}$ & 1.06 & .802 & $\begin{array}{l}.694, \\
1.60\end{array}$ & .14 & .124 & -.11 & .230 \\
\hline Data Misuse & .84 & .840 & $\begin{array}{l}.683 \\
1.36\end{array}$ & 1.03 & .851 & $\begin{array}{l}.744, \\
1.03\end{array}$ & - & - & -.22 & .003 \\
\hline $\begin{array}{c}\text { Perceived } \\
\text { Financial } \\
\text { Benefit }\end{array}$ & 1.79 & $<.001$ & $\begin{array}{l}1.33 \\
2.42\end{array}$ & .94 & .668 & $\begin{array}{l}.718 \\
1.24\end{array}$ & .35 & $<.001$ & .35 & $<.001$ \\
\hline Pseudo- $R^{2}$ & .24 & & & .10 & & & & & & \\
\hline $\begin{array}{c}\text { correctly } \\
\text { predicted (\%) }\end{array}$ & 69.6 & & & 68.5 & & & & & & \\
\hline $\mathrm{R}^{2}$ & & & & & & & .24 & & .24 & \\
\hline
\end{tabular}

Note. List-wise exclusion of cases was used, OR= Odds Ratio, $\mathrm{Cl}=90 \%$ Confidence Interval, Pseudo- $\mathrm{R}^{2}$ $=$ Nagelkerkes $\mathrm{R}^{2}$ 
4.2.3.2 Student Sample. The analyses of Ridesharing in the student sample yielded an unexpected relationship between Interpersonal Distrust $(O R=1.49, p=.026)$ and Ridesharing intention. As in the community sample, Financial Benefit was a significant predictor (OR $=1.60, p<$ .001) for Ridesharing intention. Additionally, Autonomy Loss was a significant barrier for Ridesharing intention (OR $=0.56, p=.003)$. With regard to Ridesharing behaviour, the barrier of Autonomy Loss was significant ( $O R=.57, p=.002)$ as well as the Financial Benefit $(O R=1.30, p=.016)$. For the Ridesharing neighbour-scenario only Financial Benefit $(\beta=.20, p=.001)$ was a small significant predictor. In contrast, for the internet-scenario privacy $(\beta=-.36, p<.001)$ as well as Financial Benefit $(\beta=.17, p=.002)$ were significant. The results are summarized in Table 10.

\section{Table 10}

"Ridesharing": Odds ratios on intention and behaviour and path coefficients on scenario ratings (student sample)

\begin{tabular}{|c|c|c|c|c|c|c|c|c|c|c|}
\hline & \multicolumn{6}{|c|}{ Logistic Regressions } & \multicolumn{4}{|c|}{ Path Models } \\
\hline & \multicolumn{3}{|c|}{$\begin{array}{l}\text { Intention } \\
(\mathrm{N}=265)\end{array}$} & \multicolumn{3}{|c|}{$\begin{array}{l}\text { Behaviour } \\
(N=265)\end{array}$} & \multicolumn{2}{|c|}{$\begin{array}{l}\text { Neighbour- } \\
\text { Scenario } \\
(\mathrm{N}=265)\end{array}$} & \multicolumn{2}{|c|}{$\begin{array}{l}\text { Internet- } \\
\text { Scenario } \\
(\mathrm{N}=265)\end{array}$} \\
\hline & OR & $\mathbf{p}$ & $\mathrm{Cl}$ & OR & $p$ & $\mathrm{Cl}$ & $\beta$ & $p$ & $\beta$ & $p$ \\
\hline $\begin{array}{l}\text { Autonomy } \\
\text { Loss }\end{array}$ & .56 & .003 & $\begin{array}{l}.383, \\
.826\end{array}$ & .57 & .003 & $\begin{array}{l}.399, \\
.823\end{array}$ & -.08 & .244 & -.06 & .331 \\
\hline $\begin{array}{l}\text { Privacy } \\
\text { Invasion }\end{array}$ & .75 & .134 & $\begin{array}{l}.513 \\
1.09\end{array}$ & .71 & .070 & $\begin{array}{l}.712 \\
1.14\end{array}$ & -.16 & .100 & -.36 & $<.001$ \\
\hline $\begin{array}{l}\text { Interpersonal } \\
\text { Distrust }\end{array}$ & 1.49 & .027 & $\begin{array}{l}1.05 \\
2.12\end{array}$ & 1.31 & .114 & $\begin{array}{l}.935, \\
1.84\end{array}$ & .07 & .461 & -.03 & .711 \\
\hline Data Misuse & .91 & .504 & $\begin{array}{l}.702, \\
1.19\end{array}$ & 1.14 & .301 & $\begin{array}{l}.885 \\
1.47\end{array}$ & - & - & -.08 & .208 \\
\hline $\begin{array}{c}\text { Perceived } \\
\text { Financial } \\
\text { Benefit }\end{array}$ & 1.60 & $<.001$ & $\begin{array}{l}1.28 \\
2.01\end{array}$ & 1.30 & .016 & $\begin{array}{l}1.05 \\
1.60\end{array}$ & .20 & $<.001$ & .17 & .002 \\
\hline Pseudo- $\mathrm{R}^{2}$ & .20 & & & .13 & & & & & & \\
\hline $\begin{array}{c}\text { correctly } \\
\text { predicted (\%) }\end{array}$ & 68.7 & & & 52.1 & & & & & & \\
\hline $\mathrm{R}^{2}$ & & & & & & & .08 & & .27 & \\
\hline
\end{tabular}




\section{Discussion}

Overall, the results reveal the relevance of psychological barriers for collaborative car use intentions and behaviours in general. In more detail, it shows that different barriers have different effects on different forms of P2P collaborative car use. At this point, we only discuss effects, that were significant in both samples for the same form of P2P collaborative car use.

The statistical tests confirmed a 5-factor solution with the final scales of Autonomy Loss, Personal Space Invasion, Interpersonal Distrust, Data Misuse, and Financial Benefits. In both samples and over all three forms of P2P collaborative car use, the final scales correlated mostly weakly to moderately with the general collaborative mobility constructs based on the TPB and NAM (Schwartz, 1977; Ajzen, 1991). The correlations in the expected directions, indicate nomological validity of the psychological barrier scales.

\subsection{Relevance of Psychological Barriers to Intentions and Behaviour}

Lending one's own car can be associated with the feeling of personal space invasion, as people often perceive their own car as an extension of their personal space (Fraine et al., 2007). In general, the results support this assumption. The more this potential threat of privacy is perceived, the less likely it is that the renting of one's own car to another private person is intended. Also, the stronger people assess this potential Privacy Invasion, the lower they report their willingness to use Lending To options. This is also the case if the contact to the other participants is established via the internet or neighborhood.

When renting a car from a stranger, Privacy Invasion and Data Misuse are both relevant barriers, but only if the rental is organised via the internet. This shows that establishing a trustworthy relationship to a stranger might be more difficult via the internet compared to an offline face-to-face setting. There is some evidence that people approach online negotiations with less initial trust and are generally less satisfied by the outcomes when compared to face-to-face negotiations (Naquin and 
Paulson, 2003). Accordingly, renting a car from a stranger with whom one only communicated online might increase the perception of Privacy Invasion, compared to the rental of a car from a betterknown person. Similarly, the psychological barrier of Data Misuse might be higher during the organisation via the internet, when personal data must be shared with strangers. Out of the three forms of P2P collaborative car use, Renting From probably demands sharing the highest amount of personal data especially when the rental is organised via the internet. The higher the concern regarding insufficient data security on internet platforms that are used to organise P2P collaborative car use is, the lower is the willingness to use Renting To options as suggested in the scenario. Therefore, data privacy concerns might especially be a barrier for the rental of a car from another private person over an online platform. A post-hoc explanation could be that the rental of a car is connected with a payment process and people may be more concerned about data security when sensitive payment data is processed. This concern may be higher the less experienced people are with online transactions.

For ridesharing, Autonomy Loss was negatively related to self-reported behaviour in both samples. This result is consistent because in contrast to the other forms of P2P collaborative car use, the collective sharing of rides in every-day life is connected with a high organisational effort and high willingness to compromise. While renting a car from or lending a car to another person involves primarily arrangements regarding times to hand over the car, Ridesharing demands further organization, for instance negotiating routes, places to pick up and get out as well as actually driving together in one car. These restrictions and limitations of personal options are likely to increase the subjective experienced Autonomy Loss.

Consistently over almost all analyses and forms of P2P collaborative car use, financial benefits were positively related to $\mathrm{P} 2 \mathrm{P}$ collaborative car use intentions and behaviours. The same applies for the willingness to use different forms of P2P collaborative car use, which were assessed by the scenarios. Economic considerations are assumed to be the most important motives for collaborative car use (Duncan, 2011; Luca and Di Pace, 2015). The results of the present study 
therefore constitute a replication of previous results and confirm the relevance of economic considerations for the specific behaviours Lending to, Renting From, and Ridesharing.

There were no relationships between Interpersonal Distrust and P2P collaborative car use. This is contrary to our expectations and multiple studies that showed trust to be a key factor in different forms of sharing economies (ter Huurne et al., 2017). The lack of this connection can possibly be attributed to an insufficient operationalization of Interpersonal Distrust. For example, aspects that are relevant for trust, such as the circumstances of the sharing situation, especially reputation, perceived risk, and interaction experience were not considered. It is also possible that conceptual differences between trust and distrust exist. Despite these limitations, the results showed significant bivariate correlations between Interpersonal Distrust and different forms of P2P collaborative car use.

Among the barriers, there were high positive intercorrelations between Privacy Invasion and Interpersonal Distrust, suggesting an overlap between those dimensions. From a theoretical perspective, Privacy Invasion and Interpersonal Distrust are two distinct constructs. In the context of P2P collaborative car use, Privacy Invasion refers to a violation of personal space (Altman, 1975) by unwanted activities of strangers in one's own private car. Interpersonal Distrust includes a lack of trust in the goodwill as well as the reliability of other participants and aspects of personal safety in the context of collaborative car use. One post-hoc explanation for the high statistical correlation of Privacy Invasion and Interpersonal Distrust is that our operationalizations of both barriers emphasize direct or indirect social interaction with strangers. 


\subsection{Limitations and Future Studies}

In general, both samples are non-representative and therefore no conclusions for other population groups and regions can be made. Furthermore, due to the correlative design of the study, no causal conclusions can be drawn. Future studies should use quasi-experiemental designs and manipulate single psychological barriers to investigate relationships between the psychological barriers and collaborative car use. Another limitation is the use of hypothetical scenarios without systematic variation of the scenario characteristics. The experimental vignette methodology (Aguinis and Bradley, 2014) or conjoint analysis (Lohrke et al., 2010) would be appropriate to estimate the exact strength of characteristics, such as online communication versus face-to-face communication.

Because the present study focused on three specific forms of P2P collaborative car use, the designed items are very specific and a limited explanatory power for other forms of collaborative mobility behaviour can be expected. It therefore remains an open question if the identified psychological barriers are also relevant for other forms of collaborative mobility behaviours such as bike sharing for instance. Future studies could use the present questionnaire as a useful orientation for the formulation of questionnaires and investigate the psychological barriers in other sharing contexts. We further used cross-sectional self-reports, not only to assess the psychological barriers, but also to measure mobility behaviours. This is an adequate and commonly used approach, but it can still involve validity problems with regard to actual behaviour (Kormos and Gifford, 2014). More exact measures of mobility behaviours and traffic modes could be realised through a combination of distance tracking and mobility diaries.

The scales showed overall acceptable to good psychometric properties. Only the scale of Autonomy Loss had a comparatively low internal consistency, specifically in the community sample. Autonomy Loss included aspects of organisational effort, for Ridesharing the loss of vehicle control as well as a general loss of personal freedom. In the CFAs for Renting To and Ridesharing, the different aspects vary considerably in their relationship to the latent factor Autonomy Loss. In future investigations, organisational effort and loss of personal freedom could possibly be investigated as 
separate factors. Furthermore, additional variables should be considered in future studies. For example, we controlled for car ownership but did not consider the psychological need for car ownership as an additional factor. A strong psychological need for car ownership can be a strong factor that prevents people from making use of Mobility as a Service (MaaS) software options (Alonso-González et al., 2020).

The present study showed no connection between Interpersonal Distrust and collaborative car use and intentions, but a strong relationship between Interpersonal Distrust and Privacy Invasion. To investigate interactions and differences between those barriers a more differentiated examination of Interpersonal Distrust could be considered, for example by differentiating between particularized, generalized and community trust (Wollebaek et al., 2012). We included action theorybased variables to evaluate nomological validity of the psychological barriers but did not include those variables in the models. Future studies should include those variables to compare the predictive strength of the psychological barriers in comparison to other predictors such as personal ecological norms, attitudes and PBC. Environmentalist identity was found to be relevant for the willingness to offer carpooling (Hartl et al., 2020), pointing out to the relevance of environmentally related motivations for collaborative car use that should be considered. In addition to these psychological variables, interactions of psychological barriers with trip characteristics and weather conditions (Tahmasseby et al., 2016) and lifestyle-characteristics (Vinayak et al., 2018) should also be examined.

Furthermore, future research can address questions regarding the balance between financial incentives and perceived barriers. In this regard, a deeper analysis of willingness to pay would seem promising to identify the extent of perceived financial benefits that are needed to significantly lower the psychological barriers. Another question that should be addressed is the evaluation of organisational characteristics that promote or inhibit the overcoming of psychological barriers. Specifically interesting for collaborative car use are mobility cooperatives because they can offer a reliable availability of different modes of transport without the need of owning a vehicle. 


\subsection{Practical Implications}

With regard to the goal of increasing the occupancy rate of cars the approach of psychological barriers can be used to enhance the acceptance and use of collaborative car use. The following practical implications and strategies can be addressed to decision makers of commercial mobility service providers as well as stakeholders in corporate mobility management. Because the local government depends strongly on political conditions we do not address this potential target group.

\subsubsection{Privacy Invasion: Encourage Personal Communication}

The problem of perceived privacy invasion is difficult to address with technical solutions as it is driven by cultural and individual factors. The perceived violation of personal space is moderated by familiarity with the person, the room, and the setting in general (Hayduk, 1983). Therefore, to overcome the barrier of Privacy Invasion, the increase of familiarity is an important strategy. This might be achieved by encouraging more elaborated forms of personal communication such as faceto-face communications or video calls when organising or finding different ways to exchange personal information. In privately organised car rental, procedures could be tried out with better known people such as friends and neighbours first before the circle of participants is further expanded.

\subsubsection{Data Misuse: Enhance Transparency and Decrease Personal Data Use}

Responsible and safe handling of collected personal data of any kind from customers or peers is generally important from a security and ethical perspective (Richards and King, 2014). Profound consumer information and transparency can increase trust in data security and help overcome the barrier of Data Misuse when renting out a car. This includes (1) information regarding the used encryption technologies during data processing, (2) rights of the consumer, for example if there is a right to request the deletion of one's own data, (3) an easy to find contact address, (4) in 
the case of a foreign provider: information on how the use of data differs from national data privacy laws. However, aside from those practical measures psychological aspects should be considered. Sharing personal data as part of receiving a service can be seen as a trade-off. Therefore, it is important whether the quantity and quality of the provided personal data is perceived as adequate in relation to the services received (Morey and Schoop, 2015). Increasing perceived benefits of the service decreases concerns about Data Misuse and increases willingness to share personal data (Kim et al., 2019).

\subsubsection{Autonomy Loss: Establish long-term Ridesharing Networks}

To weaken the psychological barrier of Autonomy Loss which is specifically relevant for Ridesharing, collaborative mobility providers should focus on minimizing the organisational effort for users, while maximizing time flexibility. As it is likely that the organisational effort of Ridesharing decreases with time and experience, one aim could be to support the establishment of stable longterm Ridesharing networks. Especially in consistently recurring trips like commuting to work or regular events this can be realized easily. However, it is a great challenge to encourage people without experience of collaborative car use to try it out and experience the realistic organisational effort. For this purpose, incentives such as vouchers for free rides could be offered. Also, corporate mobility management could offer free test-rides or vehicles for employees sharing the same vehicle on their way to work. To practically reduce efforts, dependence and enhance time flexibility, providers should establish ways to inform users in real time about delays and cancellations. Additionally they should keep the number of needed apps and services as low as possible. This can also include the possibility to book and pay rides and cars as a guest without a user account. 


\subsubsection{Financial Benefits: Heighten Awareness of Real Costs}

Because our findings support that financial benefits are the most important incentive for engaging in collaborative car use (Münzel et al., 2019), it should be considered how this effect can be utilized. There are two ways to increase the effect of financial benefits. Either financial benefits can simply be increased by making collaborative car use even cheaper, or the level of awareness as to the financial difference between private car use and collaborative car use can be raised. For example, Burlando et al. (2019) found that awareness of the actual costs of private car use is low, which might be a reason why convenience and comfort were rated as more important than financial costs in the same study. Publicising and emphasizing the possible financial benefits is therefore an important strategy, for example, by showing the differences between the sum of fixed costs and out-of-pocket costs between traveling with one's own car and a shared car through trip-based accounting.

\section{Conclusions}

The present study is the first to examine systematically and comprehensively psychological barriers of collaborative car use. An important result is the development of a valid questionnaire to make these psychological barriers measureable as stated prefeernces. The developed instrument allows a differentiated assessment and evaluation of the user's perspective, which was missing up to now. Further, through this instrument it is possible to identify the barriers that are relevant for specific forms of collaborative car use. While for ridesharing the main barrier is the subjective loss of personal autonomy, the feeling of privacy invasion is especially relevant for the lending of one's car to others. The concern of data misuse is strong, when a car is rented via internet. The results demonstrate the need of a differentiated analysis of collaborative car use practices from the user's perspective. Furthermore, the results can be applied to develop tailored interventions to overcome psychological barriers. 
PSYCHOLOGICAL BARRIERS TO PEER-TO-PEER COLLABORATIVE CAR USE

Funding: This work was supported by the Ministry of Culture and Science of the State of North RhineWestphalia (MKL), Germany [grant number 005-1703-0007] 


\section{References}

Abrahamse, W., Steg, L., Gifford, R., Vlek, C., 2009. Factors influencing car use for commuting and the intention to reduce it: A question of self-interest or morality? Transportation Research Part F: Traffic Psychology and Behaviour 12, 317-324.

Aguinis, H., Bradley, K.J., 2014. Best practice recommendations for designing and implementing experimental Vignette Methodology Studies. Organizational Research Methods 17, 351-371.

Ajzen, I., 1991. The Theory of Planned Behavior. Organizational Behavior and Human Decision Processes 50, 179-211.

Ajzen, I., 2006. Constructing a TPB questionnaire: Conceptual and methodological considerations. https://pdfs.semanticscholar.org/0574/b20bd58130dd5a961f1a2db10fd1fcbae95d.pdf.

Alonso-González, M.J., Hoogendoorn-Lanser, S., van Oort, N., Cats, O., Hoogendoorn, S., 2020. Drivers and barriers in adopting Mobility as a Service (MaaS) - A latent class cluster analysis of attitudes. Transportation Research Part A: Policy and Practice 132, 378-401.

Altman, I., 1975. The environment and social behavior: privacy, personal space, territory, crowding. Brooks/Cole, Monterey, CA.

Bachmann, F., Hanimann, A., Artho, J., Jonas, K., 2018. What drives people to carpool? Explaining carpooling intention from the perspectives of carpooling passengers and drivers. Transportation Research Part F: Traffic Psychology and Behaviour 59, 260-268.

Ballús-Armet, I., Shaheen, S.A., Clonts, K., Weinzimmer, D., 2014. Peer-to-peer carsharing: Exploring public perception and market characteristics in the San Francisco Bay Area, California. Transportation Research Record: Journal of the Transportation Research Board 2416, 27-36.

Bamberg, S., Ajzen, I., Schmidt, P., 2003. Choice of travel mode in the Theory of Planned Behavior: The roles of past behavior, habit, and reasoned action. Basic and Applied Social Psychology 25, 175-187.

Bamberg, S., Hunecke, M., Blöbaum, A., 2007. Social context, personal norms and the use of public transportation: Two field studies. Journal of Environmental Psychology 27(3), 190-203.

Bossauer, P., Neifer, T., Stevens, G., Pakusch, C., 2020. Trust versus privacy: Using connected car data in peer-to-peer carsharing, in: Proceedings of the $2020 \mathrm{CHI}$ Conference on Human Factors in Computing Systems. ACM, Honolulu HI USA, pp. 1-13.

Burlando, C., Ivaldi, E., Parra Saiani, P., Penco, L., 2019. To own or not to own? Car ownership and consumer awareness: Evidence from an Italian survey. Research in Transportation Business \& Management 33, 100435.

Chng, S., Abraham, C., White, M.P., Hoffmann, C., Skippon, S., 2018. Psychological theories of car use: An integrative review and conceptual framework. Journal of Environmental Psychology 55, 2333.

Cohen-Blankshtain, G., Rotem-Mindali, O., 2016. Key research themes on ICT and sustainable urban mobility. International Journal of Sustainable Transportation 10, 9-17.

Dill, J., McNeil, N., Howland, S., 2019. Effects of peer-to-peer carsharing on vehicle owners' travel behavior. Transportation Research Part C: Emerging Technologies 101, 70-78.

Duncan, M., 2011. The cost saving potential of carsharing in a US context. Transportation 38, 363382.

Evans, G.W., Wener, R.E., 2007. Crowding and personal space invasion on the train: Please don't make me sit in the middle. Journal of Environmental Psychology 27, 90-94.

Fraine, G., Smith, S.G., Zinkiewicz, L., Chapman, R., Sheehan, M., 2007. At home on the road? Can drivers' relationships with their cars be associated with territoriality? Journal of Environmental Psychology 27, 204-214. 
Furuhata, M., Dessouky, M., Ordóñez, F., Brunet, M.-E., Wang, X., Koenig, S., 2013. Ridesharing: The state-of-the-art and future directions. Transportation Research Part B: Methodological 57, 28-46.

Gardner, B., Abraham, C., 2008. Psychological correlates of car use: A meta-analysis. Transportation Research Part F: Traffic Psychology and Behaviour 11, 300-311.

Gardner, B., Abraham, C., 2010. Going green? Modeling the impact of environmental concerns and perceptions of transportation alternatives on decisions to drive. Journal of Applied Social Psychology 40, 831-849.

Gifford, R., 2011. The dragons of inaction: psychological barriers that limit climate change mitigation and adaptation. The American psychologist 66, 290-302.

Groth, S., 2019. Multimodal divide: Reproduction of transport poverty in smart mobility trends. Transportation Research Part A: Policy and Practice 125, 56-71.

Hartl, B., Kamleitner, B., Holub, S., 2020. Take me on a ride: The role of environmentalist identity for carpooling. Psychology \& marketing 37, 663-676.

Haustein, S., Hunecke, M., 2007. Reduced use of environmentally friendly modes of transportation caused by perceived mobility necessities: An extension of the Theory of Planned Behavior. Journal of Applied Social Psychology 37, 1856-1883.

Hayduk, L.A., 1983. Personal space: Where we now stand. Psychological bulletin 94, 293-335.

Heath, Y., Gifford, R., 2002. Extending the Theory of Planned Behavior: Predicting the use of public transportation. Journal of Applied Social Psychology 32, 2154-2189.

Hoffmann, C., Abraham, C., White, M.P., Ball, S., Skippon, S.M., 2017. What cognitive mechanisms predict travel mode choice? A systematic review with meta-analysis. Transport Reviews 37, 631652.

Hu, L.-t., Bentler, P.M., 1999. Cutoff criteria for fit indexes in covariance structure analysis: Conventional criteria versus new alternatives. Structural Equation Modeling: A Multidisciplinary Journal 6, 1-55.

Hunecke, M., Haustein, S., Grischkat, S., Böhler, S., 2007. Psychological, sociodemographic, and infrastructural factors as determinants of ecological impact caused by mobility behavior. Journal of Environmental Psychology 27, 277-292.

ifmo, 2016. Carsharing 2025 - Nische oder Mainstream?, München. https://www.ifmo.de/files/publications_content/2016/ifmo_2016_Carsharing_2025_de.pdf.

Kim, D., Park, K., Park, Y., Ahn, J.-H., 2019. Willingness to provide personal information: Perspective of privacy calculus in loT services. Computers in Human Behavior 92, 273-281.

Kormos, C., Gifford, R., 2014. The validity of self-report measures of proenvironmental behavior: A meta-analytic review. Journal of Environmental Psychology 40, 359-371.

Kumar, P., Morawska, L., Birmili, W., Paasonen, P., Hu, M., Kulmala, M., Harrison, R.M., Norford, L., Britter, R., 2014. Ultrafine particles in cities. Environment international 66, 1-10.

Lanzini, P., Khan, S.A., 2017. Shedding light on the psychological and behavioral determinants of travel mode choice: A meta-analysis. Transportation Research Part F: Traffic Psychology and Behaviour 48, 13-27.

Lohrke, F.T., Holloway, B.B., Woolley, T.W., 2010. Conjoint analysis in entrepreneurship research: A review and research agenda. Organizational Research Methods 13, 16-30.

Lois, D., López-Sáez, M., 2009. The relationship between instrumental, symbolic and affective factors as predictors of car use: A structural equation modeling approach. Transportation Research Part A: Policy and Practice 43, 790-799.

Luca, S. de, Di Pace, R., 2015. Modelling users' behaviour in inter-urban carsharing program: A stated preference approach. Transportation Research Part A: Policy and Practice 71, 59-76. 
Morey, T., Schoop, A., 2015. Customer data: Designing for transparency and trust. Harvard Business Review 93, 96-105.

Münzel, K., Piscicelli, L., Boon, W., Frenken, K., 2019. Different business models - different users? Uncovering the motives and characteristics of business-to-consumer and peer-to-peer carsharing adopters in The Netherlands. Transportation Research Part D: Transport and Environment 73, 276-306.

Naquin, C.E., Paulson, G.D., 2003. Online bargaining and interpersonal trust. The Journal of applied psychology 88, 113-120.

Nordlund, A.M., Garvill, J., 2003. Effects of values, problem awareness, and personal norm on willingness to reduce personal car use. Journal of Environmental Psychology 23, 339-347.

Paundra, J., Rook, L., van Dalen, J., Ketter, W., 2017. Preferences for car sharing services: Effects of instrumental attributes and psychological ownership. Journal of Environmental Psychology 53, 121-130.

Pham, T.Q., Nakagawa, C., Shintani, A., Ito, T., 2015. Evaluation of the effects of a personal mobility vehicle on multiple pedestrians using personal space. IEEE Transactions on Intelligent Transportation Systems 16, 2028-2037.

Pronello, C., Gaborieau, J.-B., 2018. Engaging in pro-environment travel behaviour research from a psycho-social perspective: A review of behavioural variables and theories. Sustainability 10, 2412.

Ramos, É.M.S., Bergstad, C.J., Nässén, J., 2020. Understanding daily car use: Driving habits, motives, attitudes, and norms across trip purposes. Transportation Research Part F: Traffic Psychology and Behaviour 68, 306-315.

Richards, N.M., King, J.H., 2014. Big data ethics. Wake Forest Law Review 49, 393-432.

Rosseel, Y., 2012. lavaan : An R package for structural equation modeling. J. Stat. Soft. 48.

Sanguinetti, A., Kurani, K., Ferguson, B., 2018. Is it ok to get in a car with a stranger: Risks and benefits of ride-pooling in shared automated vehicles.

https://escholarship.org/uc/item/1cb6n6r9. Accessed 26 August 2020.

Sarriera, J.M., Álvarez, G.E., Blynn, K., Alesbury, A., Scully, T., Zhao, J., 2017. To share or not to share: Investigating the social aspects of dynamic ridesharing. Transportation Research Record: Journal of the Transportation Research Board 2605, 109-117.

Schaefers, T., 2013. Exploring carsharing usage motives: A hierarchical means-end chain analysis. Transportation Research Part A: Policy and Practice 47, 69-77.

Schwartz, S.H., 1977. Normative influences on altruism. In: Advances in Experimental Social Psychology Volume 10, vol. 10. Elsevier, pp. 221-279.

Shaheen, S.A., Mallery, M.A., Kingsley, K.J., 2012. Personal vehicle sharing services in North America. Research in Transportation Business \& Management 3, 71-81.

Smith, G., Sochor, J., Karlsson, I.M., 2020. Intermediary MaaS Integrators: A case study on hopes and fears. Transportation Research Part A: Policy and Practice 131, 163-177.

Stanley, J., Ellison, R., Loader, C., Hensher, D., 2018. Reducing Australian motor vehicle greenhouse gas emissions. Transportation Research Part A: Policy and Practice 109, 76-88.

Steg, L., 2005. Car use: lust and must. Instrumental, symbolic and affective motives for car use. Transportation Research Part A: Policy and Practice 39, 147-162.

Tahmasseby, S., Kattan, L., Barbour, B., 2016. Propensity to participate in a peer-to-peer socialnetwork-based carpooling system. J. Adv. Transp. 50, 240-254.

ter Huurne, M., Ronteltap, A., Corten, R., Buskens, V., 2017. Antecedents of trust in the sharing economy: A systematic review. Journal of Consumer Behaviour 16, 485-498. 
Thomas, G.O., Walker, I., Musselwhite, C., 2014. Grounded Theory analysis of commuters discussing a workplace carbon-reduction target: Autonomy, satisfaction, and willingness to change behaviour in drivers, pedestrians, bicyclists, motorcyclists, and bus users. Transportation Research Part F: Traffic Psychology and Behaviour 26, 72-81.

Tussyadiah, I., 2015. An exploratory study on drivers and deterrents of collaborative consumption in travel. In: Tussyadiah, I., Inversini, A. (Eds.) Information and Communication Technologies in Tourism 2015. Springer International Publishing, Cham, pp. 817-830.

Urry, J., 2004. The 'system' of automobility. Theory, Culture \& Society 21, 25-39.

Verplanken, B., Aarts, H., van Knippenberg, A., Moonen, A., 1998. Habit versus planned behaviour: a field experiment. The British journal of social psychology 37 (Pt 1), 111-128.

Vinayak, P., Dias, F.F., Astroza, S., Bhat, C.R., Pendyala, R.M., Garikapati, V.M., 2018. Accounting for multi-dimensional dependencies among decision-makers within a generalized model framework: An application to understanding shared mobility service usage levels. Transport Policy 72, 129137.

Wang, H., Yang, H., 2019. Ridesourcing systems: A framework and review. Transportation Research Part B: Methodological 129, 122-155.

Wollebaek, D., Lundåsen, S.W., Trägårdh, L., 2012. Three forms of interpersonal trust: Evidence from swedish municipalities. Scandinavian Political Studies 35, 319-346.

World Health Organization, 2018. COP24 special report: Health and Climate Change. World Health Organization, Geneva, 74 pp. Accessed 1 September 2020.

Zhang, M., Zhao, P., Qiao, S., 2020. Smartness-induced transport inequality: Privacy concern, lacking knowledge of smartphone use and unequal access to transport information. Transport Policy 99, 175-185. 


\section{Appendix}

\section{Appendix A}

Sample Characteristics

\begin{tabular}{|c|c|c|c|c|}
\hline \multirow[t]{2}{*}{ Variable } & \multicolumn{2}{|c|}{ Sample 1: Bottrop ${ }^{a}$} & \multicolumn{2}{|c|}{ Sample 2: undergraduate students ${ }^{b}$} \\
\hline & $n$ & $\%$ & $n$ & $\%$ \\
\hline \multicolumn{5}{|l|}{ Gender } \\
\hline Female & 80 & 45.5 & 145 & 54.7 \\
\hline Male & 95 & 54.0 & 118 & 44.5 \\
\hline Other & 1 & 0.6 & 2 & 0.8 \\
\hline \multicolumn{5}{|l|}{ Monthly net income (Euro) } \\
\hline Less than 500 & 3 & 1.7 & 39 & 14.7 \\
\hline $501-1000$ & 5 & 2.8 & 59 & 22.3 \\
\hline $1001-1500$ & 8 & 4.5 & 38 & 14.3 \\
\hline $1501-2000$ & 16 & 9.1 & 26 & 9.8 \\
\hline $2001-2500$ & 17 & 9.7 & 30 & 11.3 \\
\hline $2501-3000$ & 34 & 19.3 & 15 & 5.7 \\
\hline $3001-3500$ & 20 & 11.4 & 18 & 6.8 \\
\hline $3501-4000$ & 15 & 8.5 & 9 & 3.4 \\
\hline More than 4000 & 39 & 22.2 & 27 & 10.2 \\
\hline Not specified & 19 & 10.8 & 4 & 1.5 \\
\hline \multicolumn{5}{|l|}{ Education } \\
\hline Elementary school & 2 & 1.1 & 0 & 0 \\
\hline Main school & 31 & 17.6 & 1 & 0.4 \\
\hline Middle school & 42 & 23.9 & 1 & 0.4 \\
\hline $\begin{array}{l}\text { Higher education entrance } \\
\text { qualification }\end{array}$ & 35 & 19.9 & 189 & 71.3 \\
\hline University Degree & 57 & 32.4 & 71 & 26.8 \\
\hline Other & 6 & 3.4 & 3 & 1.1 \\
\hline Not specified & 3 & 1.7 & 0 & 0 \\
\hline \multicolumn{5}{|l|}{ Occupation } \\
\hline Pupil & 1 & 0.6 & 0 & 0 \\
\hline Vocational training & 0 & 0 & 1 & 0.4 \\
\hline Undergraduate student & 0 & 0 & 245 & 92.5 \\
\hline employee & 95 & 54.0 & 4 & 1.5 \\
\hline Self-employed & 6 & 3.4 & 1 & 0.4 \\
\hline Unemployed & 14 & 8.0 & 2 & 0.8 \\
\hline Pensioner & 52 & 29.5 & 0 & 0 \\
\hline Other & 4 & 2.3 & 12 & 4.5 \\
\hline Not specified & 4 & 2.3 & 0 & 0 \\
\hline \multicolumn{5}{|l|}{ Place of birth } \\
\hline Germany & 171 & 97.2 & 243 & 91.7 \\
\hline Other & 5 & 2.8 & 22 & 8.3 \\
\hline \multicolumn{5}{|l|}{$\begin{array}{l}\text { Number of persons in the } \\
\text { household }\end{array}$} \\
\hline 1 & 23 & 13.1 & 47 & 17.1 \\
\hline 2 & 89 & 50.6 & 85 & 32.1 \\
\hline 3 & 32 & 18.2 & 65 & 24.5 \\
\hline 4 & 23 & 13.1 & 42 & 15.8 \\
\hline 5 & 8 & 4.5 & 16 & 6.0 \\
\hline 6 & 1 & 0.6 & 5 & 1.9 \\
\hline
\end{tabular}


PSYCHOLOGICAL BARRIERS TO PEER-TO-PEER COLLABORATIVE CAR USE

\begin{tabular}{|c|c|c|c|c|}
\hline \multirow[t]{2}{*}{ Variable } & \multicolumn{2}{|c|}{ Sample 1: Bottrop ${ }^{\text {a }}$} & \multicolumn{2}{|c|}{ Sample 2: undergraduate students } \\
\hline & $n$ & $\%$ & $n$ & $\%$ \\
\hline 7 & 0 & 0 & 2 & 0.8 \\
\hline more & 0 & 0 & 2 & 0.8 \\
\hline Not specified & 0 & 0 & 1 & 0.4 \\
\hline \multicolumn{5}{|l|}{ Number of children in household } \\
\hline 0 & 132 & 75.0 & 229 & 86.4 \\
\hline 1 & 16 & 9.1 & 22 & 8.3 \\
\hline 2 & 17 & 9.7 & 10 & 3.8 \\
\hline 3 & 6 & 3.4 & 2 & 0.8 \\
\hline 4 & 1 & 0.6 & 0 & 0 \\
\hline Not specified & 4 & 2.3 & 2 & 0.8 \\
\hline \multicolumn{5}{|l|}{ Working/studying in the same city } \\
\hline Yes & 42 & 23.9 & 146 & 55.1 \\
\hline No & 83 & 47.2 & 118 & 44.5 \\
\hline not applicable or not specified & 51 & 29.0 & 1 & 0.4 \\
\hline \multicolumn{5}{|l|}{ Number of cars in household } \\
\hline 0 & 4 & 2.3 & 63 & 23.8 \\
\hline 1 & 67 & 38.1 & 111 & 41.9 \\
\hline 2 & 91 & 51.7 & 51 & 19.2 \\
\hline 3 & 7 & 4.0 & 24 & 9.1 \\
\hline 4 & 1 & 0.6 & 9 & 3.4 \\
\hline 5 & 0 & 0 & 3 & 1.1 \\
\hline Not specified & 6 & 3.4 & 4 & 1.5 \\
\hline
\end{tabular}

Note. ${ }^{a} N=176$. Four participants did not answer their age. Participants were on average 53.3 years old $(S D=14.5) .{ }^{\text {b }} N=265$. Participants were on average 24.2 years old $(S D=4.4)$. Rounded percentage 


\section{Appendix B}

\section{List of final items}

\begin{tabular}{|c|c|c|c|}
\hline & German Original & Scale & English Translation \\
\hline \multicolumn{4}{|c|}{ GENERAL COLLABORATIVE CAR USE } \\
\hline \multirow[t]{2}{*}{ Intention } & $\begin{array}{l}\text { Im Allgemeinen ist es mein Ziel, häufig Pkws } \\
\text { gemeinschaftlich zu nutzen. }\end{array}$ & $\begin{array}{l}\text { 5-point Likert scale (1 „stimmt nicht“ [not true] to } 5 \\
\text { "stimmt sehr" [very true]) }\end{array}$ & $\begin{array}{l}\text { In general, my goal is to frequently use cars } \\
\text { collaboratively. }\end{array}$ \\
\hline & $\begin{array}{l}\text { Es ist nicht mein Ziel, häufig Pkws gemeinschaftlich zu } \\
\text { nutzen. }\end{array}$ & & $\begin{array}{l}\text { It is not my goal to frequently use cars } \\
\text { collaboratively. }\end{array}$ \\
\hline \multirow{2}{*}{$\begin{array}{l}\text { Perceived } \\
\text { Behaviour } \\
\text { Control }\end{array}$} & $\begin{array}{l}\text { Ich finde es schwer, die Wege in meinem Alltag mit } \\
\text { einem gemeinschaftlich genutzten Pkw } \\
\text { zurückzulegen. }\end{array}$ & & $\begin{array}{l}\text { I find it difficult to travel the distances in my } \\
\text { everyday life with a collaboratively used car. }\end{array}$ \\
\hline & $\begin{array}{l}\text { Wenn ich es möchte, fällt es mir leicht, meine } \\
\text { Alltagswege mit einem gemeinschaftlich genutzten } \\
\text { Pkw zurückzulegen. }\end{array}$ & & $\begin{array}{l}\text { If I want to, I can easily make my everyday journeys } \\
\text { in a collaboratively used car. }\end{array}$ \\
\hline \multirow[t]{3}{*}{ Personal Norm } & $\begin{array}{l}\text { Meine persönlichen Werte verpflichten mich dazu, aus } \\
\text { ökologischen Gründen Pkws möglichst häufig } \\
\text { gemeinschaftlich zu nutzen. }\end{array}$ & $\begin{array}{l}\text { 5-point Likert scale (1 „stimmt nicht" [not true] to } 5 \\
\text { "stimmt sehr" [very true]) }\end{array}$ & $\begin{array}{l}\text { My personal values oblige me to use cars } \\
\text { collaboratively as often as possible for ecological } \\
\text { reasons. }\end{array}$ \\
\hline & $\begin{array}{l}\text { Es ist meine persönliche Pflicht, aus ökologischen } \\
\text { Gründen möglichst häufig Pkws gemeinschaftlich zu } \\
\text { nutzen. }\end{array}$ & & $\begin{array}{l}\text { It is my personal duty to use cars collaboratively as } \\
\text { often as possible for ecological reasons. }\end{array}$ \\
\hline & $\begin{array}{l}\text { Ich fühle mich aus ökologischen Gründen persönlich } \\
\text { dazu verpflichtet, möglichst häufig Pkws } \\
\text { gemeinschaftlich zu nutzen. }\end{array}$ & & $\begin{array}{l}\text { For ecological reasons, I feel a personal obligation } \\
\text { to use cars collaboratively as often as possible. }\end{array}$ \\
\hline Social Norm & $\begin{array}{l}\text { Viele der Menschen, die mir wichtig sind, denken, ich } \\
\text { sollte möglichst häufig Pkws gemeinschaftlich nutzen. }\end{array}$ & $\begin{array}{l}\text { 5-point Likert scale (1 „stimmt nicht" [not true] to } 5 \\
\text { "stimmt sehr" [very true]) }\end{array}$ & $\begin{array}{l}\text { Many of the people who are important to me think } \\
\text { that I should use cars collaboratively as often as } \\
\text { possible. }\end{array}$ \\
\hline
\end{tabular}


Menschen, die mir nahestehen, finden es gut, wenn ich für meine Wege im Alltag Pkws gemeinschaftlich nutze.

Menschen, die mir wichtig sind, denken, ich sollte Pkws bevorzugt gemeinschaftlich anstatt alleine nutzen.

\begin{tabular}{|c|c|c|c|}
\hline Attitude & $\begin{array}{l}\text { unpraktisch - praktisch } \\
\text { kompliziert - unkompliziert } \\
\text { unangenehm - angenehm }\end{array}$ & $\begin{array}{l}\text { The words were presented as poles. In between was } \\
\text { a 5-point scale }\end{array}$ & $\begin{array}{l}\text { inconvenient - convenient } \\
\text { complicated - uncomplicated } \\
\text { unpleasant - pleasant }\end{array}$ \\
\hline \multicolumn{4}{|l|}{ LENDING TO } \\
\hline Behaviour & $\begin{array}{l}\text { Wie häufig verleihen Sie Ihren PKW an Personen } \\
\text { außerhalb Ihres Haushaltes? }\end{array}$ & 5-point scale (1 „nie“ [never] to 5 „immer“ [always]) & $\begin{array}{l}\text { How often do you lend your car to other people } \\
\text { outside your household? }\end{array}$ \\
\hline \multirow[t]{2}{*}{ Privacy Invasion } & $\begin{array}{l}\text { Mein Pkw ist für mich ein privater Raum, welchen ich } \\
\text { ungern jemand Fremdes unbeobachtet nutzen lasse. }\end{array}$ & $\begin{array}{l}\text { 5-point Likert scale (1 „stimmt nicht“ [not true] to } 5 \\
\text { "stimmt sehr" [very true]) }\end{array}$ & $\begin{array}{l}\text { My car is a private space for me, which I do not like } \\
\text { to let a stranger use unobservedly. }\end{array}$ \\
\hline & $\begin{array}{l}\text { Mein Pkw ist mein privater Raum, den ich nicht mit } \\
\text { jemand Fremdes teilen möchte. }\end{array}$ & & $\begin{array}{l}\text { My car is my private space which I do not want to } \\
\text { share with strangers. }\end{array}$ \\
\hline Data Misuse & $\begin{array}{l}\text { Ich habe kein Vertrauen in den Datenschutz von } \\
\text { Internetplattformen, die den Verleih von privaten } \\
\text { Pkws organisieren. }\end{array}$ & $\begin{array}{l}\text { 5-point Likert scale (1 „stimmt nicht“ [not true] to } 5 \\
\text { "stimmt sehr" [very true]) }\end{array}$ & $\begin{array}{l}\text { I have no trust in the privacy policy of internet } \\
\text { platforms that organise the rental of private cars. }\end{array}$ \\
\hline \multirow[t]{2}{*}{$\begin{array}{l}\text { Interpersonal } \\
\text { Distrust }\end{array}$} & $\begin{array}{l}\text { Wenn ich meinen Pkw verleihe habe ich die Sorge, dass } \\
\text { dieser beschädigt wird. }\end{array}$ & $\begin{array}{l}\text { 5-point Likert scale (1 „stimmt nicht“ [not true] to } 5 \\
\text { "stimmt sehr" [very true]) }\end{array}$ & When I lend my car, I worry that it will be damaged. \\
\hline & $\begin{array}{l}\text { Ich mache mir Sorgen, dass mein Pkw nicht pfleglich } \\
\text { behandelt wird, wenn ich ihn verleihe. }\end{array}$ & & $\begin{array}{l}\text { I worry that my car is not treated with care when I } \\
\text { lend it out. }\end{array}$ \\
\hline \multirow[t]{2}{*}{ Autonomy Loss } & $\begin{array}{l}\text { Die notwendigen Absprachen zur Organisation eines } \\
\text { privaten Pkw-Verleihs finde ich aufwendig. }\end{array}$ & $\begin{array}{l}\text { 5-point Likert scale (1 „stimmt nicht" [not true] to } 5 \\
\text { "stimmt sehr" [very true]) }\end{array}$ & $\begin{array}{l}\text { I find it organisationally complex to offer my car for } \\
\text { rental. }\end{array}$ \\
\hline & $\begin{array}{l}\text { Ich finde es organisatorisch aufwendig, mein Pkw zum } \\
\text { Verleih anzubieten. }\end{array}$ & & $\begin{array}{l}\text { I find it organizationally complex to offer my car for } \\
\text { lend. }\end{array}$ \\
\hline
\end{tabular}

People who are close to me find it good if I use cars collaboratively for my everyday ways.

People who are important to me think that I should preferably use cars collaboratively rather than alone.

inconvenient - convenient

mplicate 
Ich verliere meine Unabhängigkeit in der Nutzung meines Pkws, wenn ich diesen an andere Personen verleihe.

Es ist für mich eine persönliche Einschränkung, wenn mein Pkw mir nicht immer zur Verfügung steht.

\section{Perceived Durch den Verleih meines Pkw hätte ich finanzielle}

Financial Benefit Vorteile.

Neighbourhood Da Sie Ihren Pkw nicht immer brauchen, stellen Sie

Scenario 5-point Likert scale (1 „stimmt nicht“ [not true] to 5

"stimmt sehr" [very true])
I lose my independence regarding the use of my car if I lend it to other people.

It is a personal restriction for me if my car is not always available to me.

5-point Likert scale (1 „stimmt nicht“ [not true] to 5 By lending out my car I would have financial "stimmt sehr" [very true])

\section{advantages.}

Bitte bewerten Sie, inwiefern Sie die folgenden Szenarien in Ihrem Alltag nutzen würden. [Please evaluate to what extent you would use the following scenarios in your everyday life.] häufig, wodurch die Absprachen rund um den Pkw meist persönlich stattfinden. Im Gegenzug erhalten Sie Fahrtgeld.

5-point scale ( 1 ,keinesfalls" [certainly not] to 5 "ganz sicher" [for certain])

Internet Sie bieten Ihren Pkw auf einer Internetplattform zum

Internet

Verleih an. Sie bekommen regelmäßig kurzfristige Leihanfragen, woraufhin Sie die Verfügbarkeit Ihres Pkws angeben. Für Interessierte sind Sie über die Plattform und über Ihr Handy erreichbar. Im Schnitt verdienen Sie 30 Euro pro Tag für den Verleih Ihres Pkws.

5-point scale ( 1 „keinesfalls“ [certainly not] to 5
"ganz sicher" [for certain])

Because you do not always need your car, you regularly lend your car to a neighbouring couple. You know each other very well and see each other frequently, so the arrangements concerning the car are usually made in person. In return, you receive travel allowance.

You offer your car for rent on an internet platform You regularly receive short-term rental requests, whereupon you indicate the availability of your car. Interested persons can contact you via the platform and your mobile phone. On average, you earn 30 Euros per day for the rental of your car.

\section{RENTING FROM}

Behaviour $\quad$ Wie häufig leihen Sie einen Pkw von anderen Personen
außerhalb lhres Haushaltes aus?

Privacy Invasion Es ist mir unangenehm, einen Pkw von einer mir unbekannten Person zu nutzen, da ich damit in dessen Privatsphäre eindringe.

Es wäre mir unangenehm, mit privaten Dingen im geliehenen Pkw einer mir unbekannten Person konfrontiert zu werden.

Data Misuse Ich habe kein Vertrauen in den Datenschutz von Internetplattformen, bei denen ich mir von privat einen Pkw ausleihen kann.

\author{
5-point scale (1 „nie“ [never] to 5 „immer“ [always]) How often do you rent a car from other people \\ outside of your household? \\ 5-point Likert scale (1 „stimmt nicht“ [not true] to 5 \\ It is unpleasant for me to use a car of an unknown \\ person, because doing this invades his/her \\ privacy. \\ It would be unpleasant for me to be confronted \\ with private things in the rented car of a person \\ unknown to me. \\ 5-point Likert scale (1 „stimmt nicht“ [not true] to 5 I have no trust in the data privacy of internet \\ "stimmt sehr" [very true]) \\ person.
}


Ich möchte keine Informationen über meine Ausleihen von Pkws auf einer Onlineplattform preisgeben.
I do not want to reveal any information about my car rentals on an online platform.

\begin{tabular}{|c|c|c|c|}
\hline $\begin{array}{l}\text { Interpersonal } \\
\text { Distrust }\end{array}$ & $\begin{array}{l}\text { Wenn ich mir einen Pkw von einer Privatperson leihe, } \\
\text { wäre ich besorgt, dass diese unzuverlässig ist. } \\
\text { Ich wäre besorgt, dass mir eine Privatperson einen Pkw } \\
\text { in einem nicht ausreichend verkehrssicheren Zustand } \\
\text { verleiht. }\end{array}$ & $\begin{array}{l}\text { 5-point Likert scale (1 „stimmt nicht" [not true] to } 5 \\
\text { "stimmt sehr" [very true]) }\end{array}$ & $\begin{array}{l}\text { If I rented a car from a private person, I would be } \\
\text { concerned that he or she might be unreliable. } \\
\text { I would be concerned that a private person would } \\
\text { lend me a car in an unsafe condition. }\end{array}$ \\
\hline Autonomy Loss & $\begin{array}{l}\text { Die Ausleihe eines privaten Pkws von einer anderen } \\
\text { Privatperson finde ich zeitaufwendig. } \\
\text { Die Absprachen zur Organisation der Ausleihe eines } \\
\text { Pkws von einer anderen Privatperson finde ich } \\
\text { aufwendig. }\end{array}$ & $\begin{array}{l}\text { 5-point Likert scale (1 „stimmt nicht" [not true] to } 5 \\
\text { "stimmt sehr" [very true]) }\end{array}$ & $\begin{array}{l}\text { I find renting a private car from another private } \\
\text { person to be time-consuming. } \\
\text { I consider the arrangements for organising the } \\
\text { rental of a car from another private person an } \\
\text { effort. }\end{array}$ \\
\hline $\begin{array}{l}\text { Perceived } \\
\text { Financial Benefit }\end{array}$ & $\begin{array}{l}\text { Ich kann Geld sparen, wenn ich für meine Wege im } \\
\text { Alltag einen Pkw von einer Privatperson leihe. }\end{array}$ & $\begin{array}{l}\text { 5-point Likert scale (1 „stimmt nicht" [not true] to } 5 \\
\text { "stimmt sehr" [very true]) }\end{array}$ & $\begin{array}{l}\text { I can save money by renting a car from a private } \\
\text { person for my everyday trips. }\end{array}$ \\
\hline $\begin{array}{l}\text { Neighbourhood } \\
\text { Scenario }\end{array}$ & $\begin{array}{l}\text { Ihr Nachbar bietet Ihnen an, seinen Pkw gelegentlich für } \\
\text { Ihre Einkaufsfahrten zu nutzen. Im Gegenzug geben } \\
\text { Sie Ihrem Nachbar hierfür anteilig Fahrtgeld. Sie } \\
\text { tauschen sich regelmäßig aus, wann der Pkw für Sie } \\
\text { zur Verfügung steht. }\end{array}$ & $\begin{array}{l}\text { Bitte bewerten Sie, inwiefern Sie die folgenden } \\
\text { Angebote in Ihrem Alltag nutzen würden. Würden } \\
\text { Sie dieses Angebot nutzen? [Please evaluate to } \\
\text { what extent you would use the following offers in } \\
\text { your everyday life. Would you use this offer?] } \\
\text { 5-point scale ( } 1 \text { „keinesfalls“ [certainly not] to } 5 \\
\text { "ganz sicher" [for certain]) }\end{array}$ & $\begin{array}{l}\text { Your neighbour offers you to use his/her car } \\
\text { occasionally for your shopping trips. In return, } \\
\text { you give proportionate travel allowance to your } \\
\text { neighbour. You regularly communicate with each } \\
\text { other regarding when the car is available for you. }\end{array}$ \\
\hline $\begin{array}{l}\text { Internet } \\
\text { Scenario }\end{array}$ & $\begin{array}{l}\text { Sie informieren sich auf einer Internetplattform über } \\
\text { das Ausleihen eines Pkws von privat. Sie finden eine } \\
\text { Person in Ihrem Stadtteil, die viele positive } \\
\text { Bewertungen von anderen Nutzern bekommen hat. } \\
\text { Sie rufen den Anbieter an, um einen Termin zu } \\
\text { vereinbaren. Der Abholort ist für Sie fußläufig zu } \\
\text { erreichen und der Pkw kostet } 30 \text { Euro pro Tag. }\end{array}$ & $\begin{array}{l}\text { 5-point scale (1 „keinesfalls“ [certainly not] to } 5 \\
\text { „ganz sicher“ [for certain]) }\end{array}$ & $\begin{array}{l}\text { You inform yourself on an internet platform about } \\
\text { renting a car from a private person. You find a } \\
\text { person in your district who has many positive } \\
\text { reviews from other users. You call this person to } \\
\text { make an appointment. The pickup location is } \\
\text { within walking distance from you. The car costs } \\
\text { you } 30 \text { Euro per day. }\end{array}$ \\
\hline \multicolumn{4}{|l|}{ RIDESHARING } \\
\hline Behaviour & $\begin{array}{l}\text { Nutzen Sie Fahrgemeinschaften für Ihren Arbeits- } \\
\text { /Ausbildungsweg? }\end{array}$ & 5-point scale (1 „nie“ [never] to 5 „immer“ [always]) & $\begin{array}{l}\text { Do you use carpooling for your way to } \\
\text { work/training? }\end{array}$ \\
\hline
\end{tabular}




\begin{tabular}{|c|c|c|c|}
\hline Privacy Invasion & $\begin{array}{l}\text { Mit jemand mir Unbekanntes in einem Pkw zu sitzen, } \\
\text { empfinde ich als unangenehm. } \\
\text { Während einer gemeinsamen Pkw-Fahrt mit } \\
\text { unbekannten Personen, fühle mich in meiner } \\
\text { Privatsphäre eingeschränkt. }\end{array}$ & $\begin{array}{l}\text { 5-point Likert scale (1 „stimmt nicht“ [not true] to } 5 \\
\text { "stimmt sehr" [very true]) }\end{array}$ & $\begin{array}{l}\text { During a shared car ride with unknown persons, I } \\
\text { feel restricted in my privacy. }\end{array}$ \\
\hline \multirow[t]{2}{*}{ Data Misuse } & $\begin{array}{l}\text { Ich möchte keine Informationen über meine Pkw- } \\
\text { Fahrten und Mitfahrten über eine Onlineplattform } \\
\text { zugänglich machen. }\end{array}$ & $\begin{array}{l}\text { 5-point Likert scale (1 „stimmt nicht“ [not true] to } 5 \\
\text { "stimmt sehr" [very true]) }\end{array}$ & $\begin{array}{l}\text { I do not want to provide information about my car } \\
\text { trips and ride-sharing via an online platform. }\end{array}$ \\
\hline & $\begin{array}{l}\text { Ich habe kein Vertrauen in den Datenschutz von } \\
\text { Internetplattformen, die Fahrgemeinschaften } \\
\text { organisieren. }\end{array}$ & & $\begin{array}{l}\text { I have no trust in the data privacy of internet } \\
\text { platforms that organise Ridesharing. }\end{array}$ \\
\hline \multirow[t]{2}{*}{$\begin{array}{l}\text { Interpersonal } \\
\text { Distrust }\end{array}$} & $\begin{array}{l}\text { Ich fühle mich unsicher, wenn ich während einer } \\
\text { Fahrgemeinschaft mit Fremden in einem Pkw sitze. }\end{array}$ & $\begin{array}{l}\text { 5-point Likert scale (1 „stimmt nicht“ [not true] to } 5 \\
\text { "stimmt sehr" [very true]) }\end{array}$ & $\begin{array}{l}\text { I feel unsafe sitting in a car while Ridesharing with } \\
\text { strangers. }\end{array}$ \\
\hline & $\begin{array}{l}\text { Bei Fahrgemeinschaften mit fremden Personen habe ich } \\
\text { Angst vor Übergriffen. }\end{array}$ & & $\begin{array}{l}\text { When carpooling with strangers I am afraid of } \\
\text { assaults. }\end{array}$ \\
\hline \multirow[t]{4}{*}{ Autonomy Loss } & $\begin{array}{l}\text { Ich finde den Organisationsaufwand, welcher mit einer } \\
\text { Fahrgemeinschaft verbunden ist, sehr zeitaufwendig }\end{array}$ & $\begin{array}{l}\text { 5-point Likert scale (1 „stimmt nicht“ [not true] to } 5 \\
\text { "stimmt sehr" [very true]) }\end{array}$ & $\begin{array}{l}\text { I find the organisational effort involved in } \\
\text { carpooling very time-consuming. }\end{array}$ \\
\hline & $\begin{array}{l}\text { Die notwendigen Absprachen zur Organisation einer } \\
\text { Fahrgemeinschaft finde ich aufwendig. }\end{array}$ & & $\begin{array}{l}\text { I find the necessary arrangements to organize a } \\
\text { carpool effortful. }\end{array}$ \\
\hline & $\begin{array}{l}\text { Ich gebe nicht gerne die Steuerung eines Pkw an jemand } \\
\text { anderes ab. }\end{array}$ & & $\begin{array}{l}\text { I do not like handing over control of a car to } \\
\text { someone else. }\end{array}$ \\
\hline & $\begin{array}{l}\text { Ich fahre gerne im Pkw mit, auch wenn ich nicht selbst } \\
\text { am Steuer sitze. }\end{array}$ & & $\begin{array}{l}\text { I like to ride in the car, even if I am not in the } \\
\text { driver's seat myself. }\end{array}$ \\
\hline $\begin{array}{l}\text { Perceived } \\
\text { Financial Benefit }\end{array}$ & Mit einer Fahrgemeinschaft kann ich Geld sparen. & & I can save money through Ridesharing. \\
\hline $\begin{array}{l}\text { Internet } \\
\text { Scenario }\end{array}$ & $\begin{array}{l}\text { Sie sehen auf einer Internetplattform eine Annonce für } \\
\text { eine Fahrgemeinschaft für Ihren Arbeitsweg und } \\
\text { melden sich bei der betreffenden Person. Sie fahren } \\
\text { von nun an dreimal in der Woche bei der Person mit. } \\
\text { Sie werden von zu Hause abgeholt und auch } \\
\text { zurückgebracht. Sie beteiligen sich an den Fahrtkosten } \\
\text { der Fahrerin/des Fahrers. }\end{array}$ & $\begin{array}{l}\text { Bitte bewerten Sie, inwiefern Sie die folgenden } \\
\text { Angebote in Ihrem Alltag nutzen würden. Würden } \\
\text { Sie dieses Angebot nutzen? [Please evaluate to } \\
\text { what extent you would use the following offers in } \\
\text { your everyday life. Would you use this offer?] }\end{array}$ & $\begin{array}{l}\text { On an internet platform you see an offer for a } \\
\text { carpool for your way to work and contact the } \\
\text { person. From now on, you will ride with the } \\
\text { person three times a week. You are picked up } \\
\text { from your home and brought back. You } \\
\text { contribute to the driver's travel costs. }\end{array}$ \\
\hline
\end{tabular}


5 -point scale ( 1 ,keinesfalls“ [certainly not] to 5

"ganz sicher" [for certain])

Neighbourhood Ihre Nachbarin fährt an jedem Morgen ihre zwei Kinder

Scenario

zur Schule und holt sie am Nachmittag wieder ab. Sie

bietet Ihnen an, Sie mitzunehmen, da Ihre

Arbeitsstelle sich in der Nähe der Schule befindet.

Gelegentlich müssen Sie eine halbe Stunde eher als

gewöhnlich los oder werden etwas später abgeholt,

da sich Ihre Arbeitszeiten nicht vollständig mit den

Schulzeiten decken.

\section{5-point scale (1 „,keinesfalls“ [certainly not] to 5} „ganz sicher" [for certain])
Your neighbour drives her two children to schoo every morning and picks them up again in the afternoon. She offers to give you a ride, since your place of work is close to the school.

Occasionally you may need to leave half an hour earlier than usual or you may be picked up a little later because your working hours do not fully coincide with the school hours. 


\section{AUTONOMY LOSS, PRIVACY INVASION, AND DATA MISUSE IN COLLABORATIVE CAR USE}

\section{Appendix C}

\section{Table C1}

Lending To: Descriptive Data and Intercorrelations of the Scales (Community Sample)

\begin{tabular}{|c|c|c|c|c|c|c|c|c|c|c|c|c|c|c|c|c|c|c|}
\hline & & $\mathrm{M}$ & $S D$ & $\alpha$ & 1 & 2 & 3 & 4 & 5 & 6 & 7 & 8 & 9 & 10 & 11 & 12 & 13 & 14 \\
\hline 1 & Privacy Invasion & 3.99 & 1.24 & .86 & 1 & & & & & & & & & & & & & \\
\hline 2 & Data Misuse & 3.40 & 1.40 & - & $.21^{* * *}$ & 1 & & & & & & & & & & & & \\
\hline 3 & $\begin{array}{l}\text { Interpersonal } \\
\text { Distrust }\end{array}$ & 4.10 & 1.09 & .83 & $.72^{* * *}$ & $.21^{* *}$ & 1 & & & & & & & & & & & \\
\hline 4 & Autonomy Loss & 4.00 & 0.83 & .60 & $.44^{* * *}$ & .07 & $.30^{* * *}$ & 1 & & & & & & & & & & \\
\hline 5 & $\begin{array}{c}\text { Perceived } \\
\text { Financial Benefit }\end{array}$ & 2.04 & 1.02 & & -.11 & -.12 & $-.21^{* * *}$ & $-.25^{* *}$ & 1 & & & & & & & & & \\
\hline 6 & $\begin{array}{c}\text { Intention } \\
\text { (Dichotomized) }\end{array}$ & - & - & - & $-.51^{* * *}$ & $-.28^{* * *}$ & $-.40^{* * *}$ & $-.26^{* * *}$ & $-.18^{*}$ & 1 & & & & & & & & \\
\hline 7 & $\begin{array}{c}\text { Behaviour } \\
\text { (Dichotomized) }\end{array}$ & - & - & - & $-.30^{* * *}$ & -.08 & $-.22^{* *}$ & -.13 & .01 & $.28^{* * *}$ & 1 & & & & & & & \\
\hline 8 & $\begin{array}{l}\text { Neighbourhood } \\
\text { Scenario }\end{array}$ & 2.41 & 1.23 & - & $-.60^{* * *}$ & $-.26^{* * *}$ & $-.41^{* *}$ & $-.44^{* * *}$ & $.20^{*}$ & $.44^{* * *}$ & $.38^{* * *}$ & 1 & & & & & & \\
\hline 9 & Internet Scenario & 1.70 & 0.90 & - & $-.42^{* * *}$ & $-.39^{* * *}$ & $-.29^{* * *}$ & $-.30^{* * *}$ & $.38^{* * *}$ & $.41^{* * *}$ & $.18^{*}$ & $.57^{* * *}$ & 1 & & & & & \\
\hline 10 & Intention & 2.10 & 1.01 & .74 & $-.44^{* * *}$ & $-.22^{* * *}$ & $-.31^{* * *}$ & $-.39^{* * *}$ & .15 & $.41^{* * *}$ & $.22^{* *}$ & $.47^{* * *}$ & $.35^{* * *}$ & 1 & & & & \\
\hline 11 & $\begin{array}{c}\text { Perceived } \\
\text { Behaviour } \\
\text { Control }\end{array}$ & 1.92 & 0.92 & .62 & $-.33^{* * *}$ & $-.19^{*}$ & $-.25^{* *}$ & $-.31^{* * *}$ & .14 & $.39^{* * *}$ & .09 & $.37^{* * *}$ & $.32^{* * *}$ & $.40^{* * *}$ & 1 & & & \\
\hline 12 & Social Norm & 1.94 & 1.01 & .85 & $-.42^{* * *}$ & -.12 & $-.33^{* * *}$ & $-.29^{* * *}$ & .04 & $.30^{* * *}$ & $.24^{* * *}$ & $.39^{* * *}$ & $.25^{* *}$ & $.61^{* * *}$ & $.40^{* * *}$ & 1 & & \\
\hline 13 & Attitude & 2.28 & 1.07 & .80 & $-.36^{* * *}$ & $-.19^{*}$ & $-.27^{* * *}$ & $-.48^{* * *}$ & $.28^{* * *}$ & $.27^{* * *}$ & $.16^{*}$ & $.40^{* * *}$ & $.27^{* * *}$ & $.59^{* * *}$ & $.51^{* * *}$ & $.47^{* * *}$ & 1 & \\
\hline 14 & Personal Norm & 2.32 & 1.17 & .91 & $-.40^{* * *}$ & $-.15^{*}$ & $-.30^{* * *}$ & $-.23^{* * *}$ & .08 & $.35^{* * *}$ & $.17^{*}$ & $.38^{* * *}$ & $.31^{* * *}$ & $.64^{* * *}$ & $.25^{* * *}$ & $.64^{* * *}$ & $.39^{* * *}$ & 1 \\
\hline 15 & $\begin{array}{c}\text { Motorization } \\
\text { degree }\end{array}$ & 0.81 & 0.31 & - & .06 & -.01 & -.11 & .10 & -.12 & .02 & .08 & -.08 & -.08 & .05 & $-.16^{*}$ & -.09 & -.05 & -.04 \\
\hline
\end{tabular}




\section{AUTONOMY LOSS, PRIVACY INVASION, AND DATA MISUSE IN COLLABORATIVE CAR USE}

Note. Variables 10-15 refer to collaborative mobility in general. Listwise case exclusion was used. Sample Size varies between $\mathrm{N}=158-172$

\section{Table C2}

Lending To: Descriptive Data and Intercorrelations of the Scales (Student Sample)

\begin{tabular}{|c|c|c|c|c|c|c|c|c|c|c|c|c|c|c|c|c|c|c|}
\hline & & $\mathrm{M}$ & $S D$ & $\alpha$ & 1 & 2 & 3 & 4 & 5 & 6 & 7 & 8 & 9 & 10 & 11 & 12 & 13 & 14 \\
\hline 1 & Privacy Invasion & 3.70 & 1.23 & .90 & 1 & & & & & & & & & & & & & \\
\hline 2 & Data Misuse & 3.11 & 1.21 & - & $.30^{* * *}$ & 1 & & & & & & & & & & & & \\
\hline 3 & $\begin{array}{l}\text { Interpersonal } \\
\text { Distrust }\end{array}$ & 4.10 & 1.04 & .86 & $.72^{* * *}$ & $.32^{* *}$ & 1 & & & & & & & & & & & \\
\hline 4 & Autonomy Loss & 3.00 & 0.86 & .72 & $.40^{* * *}$ & $.22^{* *}$ & $.36^{* * *}$ & 1 & & & & & & & & & & \\
\hline 5 & $\begin{array}{c}\text { Perceived } \\
\text { Financial Benefit }\end{array}$ & 2.90 & 1.21 & & -.10 & -.01 & .03 & -.01 & 1 & & & & & & & & & \\
\hline 6 & $\begin{array}{c}\text { Intention } \\
\text { (Dichotomized) }\end{array}$ & - & - & - & $-.44^{* * *}$ & $-.27^{* * *}$ & $-.35^{* * *}$ & $-.23^{* *}$ & .12 & 1 & & & & & & & & \\
\hline 7 & $\begin{array}{c}\text { Behaviour } \\
\text { (Dichotomized) }\end{array}$ & - & - & - & $-.14^{*}$ & .01 & -.13 & -.01 & -.01 & .04 & 1 & & & & & & & \\
\hline 8 & $\begin{array}{l}\text { Neighbourhood } \\
\text { Scenario }\end{array}$ & 3.30 & 1.11 & - & $-.38^{* * *}$ & $-.15^{*}$ & $-.32^{* *}$ & $-.30^{* * *}$ & $.25^{* * *}$ & $.39^{* * *}$ & .01 & 1 & & & & & & \\
\hline 9 & Internet Scenario & 2.37 & 0.94 & - & $-.53^{* * *}$ & $-.23^{* *}$ & $-.45^{* * *}$ & $-.20^{* *}$ & $.17^{*}$ & $.40^{* * *}$ & .05 & $.44^{* * *}$ & 1 & & & & & \\
\hline
\end{tabular}

Scales relating to collaborative car use in general

\begin{tabular}{|c|c|c|c|c|c|c|c|c|c|c|c|c|c|c|c|c|c|c|}
\hline 10 & Intention & 2.81 & 1.01 & .87 & $-.41^{* * *}$ & $-.15^{*}$ & $-.32^{* * *}$ & $-.29^{* * *}$ & .03 & $.35^{* * *}$ & .06 & $.38^{* * *}$ & $.29^{* * *}$ & 1 & & & & \\
\hline 11 & $\begin{array}{c}\text { Perceived } \\
\text { Behaviour Control }\end{array}$ & 2.31 & 0.97 & .77 & $-.23^{* * *}$ & .00 & $-.21^{* *}$ & $-.33^{* * *}$ & .02 & $.21^{* *}$ & .01 & $.27^{* * *}$ & $.18^{*}$ & $.40^{* * *}$ & 1 & & & \\
\hline 12 & Social Norm & 2.34 & 1.00 & .82 & $-.29^{* * *}$ & -.09 & $-.17^{*}$ & $-.13^{*}$ & -.00 & $.28^{* * *}$ & -.02 & $.30^{* * *}$ & $.19^{* *}$ & $.52^{* * *}$ & $.24^{* * *}$ & 1 & & \\
\hline 13 & Attitude & 3.00 & 0.99 & .72 & $-.45^{* * *}$ & $-.16^{*}$ & $-.31^{* * *}$ & $-.49^{* * *}$ & .07 & $.31^{* * *}$ & .02 & $.31^{* * *}$ & $.26^{* * *}$ & $.54^{* * *}$ & $.47^{* * *}$ & $.37^{* * *}$ & 1 & \\
\hline 14 & Personal Norm & 2.83 & 1.22 & .93 & $-.35^{* * *}$ & $-.16^{*}$ & $-.24^{* * *}$ & $-.29^{* * *}$ & .14 & $.29^{* * *}$ & .05 & $.41^{* * *}$ & $.27^{* * *}$ & $.77^{* * *}$ & $.24^{* * *}$ & $.63^{* * *}$ & $.45^{* * *}$ & 1 \\
\hline 15 & $\begin{array}{c}\text { Motorization } \\
\text { degree }\end{array}$ & 0.52 & 0.41 & - & .09 & -.04 & .07 & .08 & $-.25^{* *}$ & -.04 & -05 & -.05 & -.05 & .00 & -.09 & -.09 & -.05 & .03 \\
\hline
\end{tabular}


AUTONOMY LOSS, PRIVACY INVASION, AND DATA MISUSE IN COLLABORATIVE CAR USE

Note. Variables 10-15 refer to collaborative mobility in general. Listwise case exclusion was used. Sample Size varies between N=194-265

\section{Table C3}

Renting From: Descriptive Data and Intercorrelations of the Scales (Community Sample)

\begin{tabular}{|c|c|c|c|c|c|c|c|c|c|c|c|c|c|c|c|c|c|c|}
\hline & & $\mathrm{M}$ & $S D$ & $\alpha$ & 1 & 2 & 3 & 4 & 5 & 6 & 7 & 8 & 9 & 10 & 11 & 12 & 13 & 14 \\
\hline 1 & Privacy Invasion & 3.40 & 1.25 & .81 & 1 & & & & & & & & & & & & & \\
\hline 2 & Data Misuse & 3.71 & 1.13 & .71 & $.53^{* * *}$ & 1 & & & & & & & & & & & & \\
\hline 3 & $\begin{array}{l}\text { Interpersonal } \\
\text { Distrust }\end{array}$ & 3.57 & 1.12 & .72 & $.61^{* * *}$ & $.48^{* * *}$ & 1 & & & & & & & & & & & \\
\hline 4 & Autonomy & 3.87 & 0.94 & .67 & $.43^{* * *}$ & $.23^{* *}$ & $.41^{* * *}$ & 1 & & & & & & & & & & \\
\hline 5 & $\begin{array}{c}\text { Perceived } \\
\text { Financial Benefit }\end{array}$ & 2.30 & 1.24 & - & $-.23^{* *}$ & $-.22^{* *}$ & $-.33^{* * *}$ & $-.17^{*}$ & 1 & & & & & & & & & \\
\hline 6 & $\begin{array}{c}\text { Intention } \\
\text { (Dichotomized }\end{array}$ & - & - & - & $-.18^{*}$ & $-.15^{*}$ & -.14 & $-.20^{* *}$ & $-.19^{*}$ & 1 & & & & & & & & \\
\hline 7 & $\begin{array}{c}\text { Behaviour } \\
\text { (Dichotomized) }\end{array}$ & - & - & - & -.12 & $-.15^{*}$ & $-.16^{*}$ & $-.16^{*}$ & .08 & .06 & 1 & & & & & & & \\
\hline 8 & $\begin{array}{l}\text { Neighbourhood } \\
\text { Scenario }\end{array}$ & 2.25 & 1.12 & - & $-.30^{* * *}$ & $-.23^{* * *}$ & $-.31^{* * *}$ & $-.38^{* * *}$ & $.34^{* *}$ & $.28^{* * *}$ & $.24^{* *}$ & 1 & & & & & & \\
\hline 9 & Internet Scenario & 1.96 & 0.96 & - & $-.43^{* * *}$ & $-.41^{* * *}$ & $-.34^{* * *}$ & $-.30^{* * *}$ & $.31^{* * *}$ & $.33^{* * *}$ & .10 & $.57^{* * *}$ & 1 & & & & & \\
\hline
\end{tabular}

Scales relating to collaborative car use in general

\begin{tabular}{|c|c|c|c|c|c|c|c|c|c|c|c|c|c|c|c|c|c|c|}
\hline 10 & Intention & 2.10 & 1.01 & .74 & $-.19^{*}$ & -.12 & $-.18^{*}$ & $-.21^{* *}$ & $.29^{* * *}$ & $.20^{*}$ & .13 & $.47^{* * *}$ & $.35^{* * *}$ & 1 & & & & \\
\hline 11 & $\begin{array}{c}\text { Perceived } \\
\text { Behaviour Control }\end{array}$ & 1.92 & 0.92 & .62 & -.09 & -.11 & -.12 & $-.31^{* * *}$ & .11 & $.19^{*}$ & .07 & $.37^{* * *}$ & $.32^{* * *}$ & $.40^{* * *}$ & 1 & & & \\
\hline 12 & Social Norm & 1.94 & 1.01 & .85 & -.05 & .03 & -.13 & $-.17^{*}$ & $.21^{* *}$ & $.16^{*}$ & .13 & $.39^{* * *}$ & $.25^{* *}$ & $.61^{* * *}$ & $.40^{* * *}$ & 1 & & \\
\hline 13 & Attitude & 2.28 & 1.07 & .80 & -.11 & -.10 & -.11 & $-.29^{* * *}$ & $.24^{* *}$ & .10 & .15 & $.40^{* * *}$ & $.27^{* * *}$ & $.59^{* * *}$ & $.51^{* * *}$ & $.47^{* * *}$ & 1 & \\
\hline 14 & Personal Norm & 2.32 & 1.17 & .91 & -.14 & -.05 & -.09 & .13 & $.29^{* * *}$ & .14 & .07 & $.38^{* * *}$ & $.31^{* * *}$ & $.64^{* * *}$ & $.25^{* * *}$ & $.64^{* * *}$ & $.39^{* * *}$ & 1 \\
\hline 15 & $\begin{array}{c}\text { Motorization } \\
\text { degree }\end{array}$ & 0.81 & 0.31 & - & -.03 & .03 & .03 & .09 & -.14 & -.10 & -.04 & -.08 & -.08 & .05 & $-.16^{*}$ & -.09 & -.05 & -.04 \\
\hline
\end{tabular}

Note. Variables 10-15 refer to collaborative mobility in general. Listwise case exclusion was used. Sample Size varies between $\mathrm{N}=158-172$ 
AUTONOMY LOSS, PRIVACY INVASION, AND DATA MISUSE IN COLLABORATIVE CAR USE

\section{Table C4}

Renting from: Descriptive Data and Intercorrelations of the Scales (Student Sample)

\begin{tabular}{|c|c|c|c|c|c|c|c|c|c|c|c|c|c|c|c|c|c|c|}
\hline & & $\mathrm{M}$ & $S D$ & $\alpha$ & 1 & 2 & 3 & 4 & 5 & 6 & 7 & 8 & 9 & 10 & 11 & 12 & 13 & 14 \\
\hline 1 & Privacy Invasion & 2.91 & 1.21 & .81 & 1 & & & & & & & & & & & & & \\
\hline 2 & Data Misuse & 3.00 & 1.17 & .72 & $.41^{* * *}$ & 1 & & & & & & & & & & & & \\
\hline 3 & $\begin{array}{l}\text { Interpersonal } \\
\text { Distrust }\end{array}$ & 3.38 & 1.07 & .67 & $.52^{* * *}$ & $.44^{* * *}$ & 1 & & & & & & & & & & & \\
\hline 4 & Autonomy Loss & 3.44 & 0.94 & .74 & $.38^{* * *}$ & $.33^{* * *}$ & $.48^{* * *}$ & 1 & & & & & & & & & & \\
\hline 5 & $\begin{array}{c}\text { Perceived } \\
\text { Financial Benefit }\end{array}$ & 2.37 & 1.26 & - & $-.13^{*}$ & -.00 & $-.13^{*}$ & $-.18^{* *}$ & 1 & & & & & & & & & \\
\hline 6 & $\begin{array}{c}\text { Intention } \\
\text { (Dichotomized) }\end{array}$ & - & - & - & $-.14^{*}$ & $-.14^{*}$ & $-.19^{* *}$ & $-.23^{* * *}$ & $.34^{* *}$ & 1 & & & & & & & & \\
\hline 7 & $\begin{array}{c}\text { Behaviour } \\
\text { (Dichotomized) }\end{array}$ & - & - & - & -.07 & -.02 & $-.19^{* *}$ & $-.11^{*}$ & .07 & $.29^{* * *}$ & 1 & & & & & & & \\
\hline 8 & $\begin{array}{l}\text { Neighbourhood } \\
\text { Scenario }\end{array}$ & 3.34 & 1.08 & - & $-.20^{* *}$ & $-.25^{* * *}$ & $-.20^{* *}$ & $-.18^{* *}$ & $.24^{* * *}$ & $.23^{* * *}$ & $.12^{*}$ & 1 & & & & & & \\
\hline 9 & $\begin{array}{l}\text { Internet } \\
\text { Scenario }\end{array}$ & 2.44 & 0.99 & - & $-.32^{* * *}$ & $-.35^{* * *}$ & $-.29^{* * *}$ & $-.27^{* * *}$ & $.36^{* * *}$ & $.31^{* * *}$ & $.17^{* *}$ & $.61^{* * *}$ & 1 & & & & & \\
\hline \multicolumn{19}{|c|}{ Scales relating to collaborative car use in general } \\
\hline 10 & Intention & 2.81 & 1.01 & .87 & $-.17^{* *}$ & $-.16^{* *}$ & $-.19^{* *}$ & $-.18^{* *}$ & $.16^{*}$ & $.24^{*}$ & $.12^{*}$ & $.30^{* * *}$ & $.23^{* * *}$ & 1 & & & & \\
\hline 11 & $\begin{array}{c}\text { Perceived } \\
\text { Behaviour } \\
\text { Control }\end{array}$ & 2.31 & 0.97 & .77 & .04 & -.11 & -.12 & $-.32^{* * *}$ & $.20^{* *}$ & .11 & .05 & $.17^{* *}$ & $.18^{* *}$ & $.34^{* * *}$ & 1 & & & \\
\hline 12 & Social Norm & 2.34 & 1.00 & .82 & -.12 & -.03 & -.10 & -.08 & $.23^{* *}$ & $.21^{* *}$ & .08 & $.24^{* * *}$ & $.26^{* * *}$ & $.52^{* * *}$ & $.24^{* * *}$ & 1 & & \\
\hline 13 & Attitude & 3.00 & 0.99 & .72 & $-.18^{* *}$ & $-.14^{*}$ & $-.17^{* *}$ & $-.36^{* * *}$ & $.15^{*}$ & $.25^{* * *}$ & .11 & $.29^{* * *}$ & $.21^{* * *}$ & $.54^{* * *}$ & $.47^{* * *}$ & $.37^{* * *}$ & 1 & \\
\hline 14 & Personal Norm & 2.83 & 1.22 & .93 & $-.19^{* *}$ & $-.19^{* *}$ & $-.15^{*}$ & -11 & $.22^{* * *}$ & $.19^{* *}$ & .10 & $.32^{* * *}$ & $.27^{* * *}$ & $.77^{* * *}$ & $.24^{* * *}$ & $.63^{* * *}$ & $.45^{* * *}$ & 1 \\
\hline 15 & $\begin{array}{l}\text { Motorization } \\
\text { degree }\end{array}$ & 0.52 & 0.41 & - & .11 & $.13^{*}$ & $.20^{* *}$ & $.16^{*}$ & -.04 & -.12 & $-.15^{*}$ & $-.18^{* *}$ & $-.19^{* *}$ & .00 & -.09 & .02 & -.05 & .03 \\
\hline
\end{tabular}

Note. Variables 10-15 refer to collaborative mobility in general. Listwise case exclusion was used. Sample Size varies between $\mathrm{N}=256-265$ 
AUTONOMY LOSS, PRIVACY INVASION, AND DATA MISUSE IN COLLABORATIVE CAR USE

\section{Table C5}

Ridesharing: Descriptive Data and Intercorrelations of the Scales (Community Sample)

\begin{tabular}{|c|c|c|c|c|c|c|c|c|c|c|c|c|c|c|c|c|c|c|}
\hline & & $\mathrm{M}$ & $S D$ & $\alpha$ & 1 & 2 & 3 & 4 & 5 & 6 & 7 & 8 & 9 & 10 & 11 & 12 & 13 & 14 \\
\hline 1 & Privacy Invasion & 3.37 & 1.14 & .78 & 1 & & & & & & & & & & & & & \\
\hline 2 & Data Misuse & 3.78 & 1.13 & .77 & $.40^{* * *}$ & 1 & & & & & & & & & & & & \\
\hline 3 & $\begin{array}{l}\text { Interpersonal } \\
\text { Distrust }\end{array}$ & 2.81 & 1.19 & .74 & $.71^{* * *}$ & $.35^{* * *}$ & 1 & & & & & & & & & & & \\
\hline 4 & Autonomy Loss & 3.35 & 0.88 & .60 & $.50^{* * *}$ & $.32^{* *}$ & $.35^{* * *}$ & 1 & & & & & & & & & & \\
\hline 5 & $\begin{array}{c}\text { Perceived } \\
\text { Financial Benefit }\end{array}$ & 2.81 & 1.35 & - & $-.25^{* *}$ & -.11 & $-.21^{* *}$ & $-.35^{* * *}$ & 1 & & & & & & & & & \\
\hline 6 & $\begin{array}{l}\text { Intention } \\
\text { (Dichotomized }\end{array}$ & - & - & - & $-.19^{*}$ & -.09 & -.06 & $-.29^{* * *}$ & $-.37^{* * *}$ & 1 & & & & & & & & \\
\hline 7 & $\begin{array}{c}\text { Behaviour } \\
\text { (Dichotomized) }\end{array}$ & - & - & - & -.07 & -.03 & -.04 & $-.25^{* *}$ & .05 & $.32^{* * *}$ & 1 & & & & & & & \\
\hline 8 & $\begin{array}{l}\text { Neighbourhood } \\
\text { Scenario }\end{array}$ & 2.45 & 1.16 & - & $-.19^{*}$ & -.11 & -.07 & $-.36^{* * *}$ & $.42^{* * *}$ & $.46^{* * *}$ & $.22^{* *}$ & 1 & & & & & & \\
\hline \multirow[t]{2}{*}{9} & Internet Scenario & 2.39 & 1.20 & - & $-.43^{* * *}$ & $-.28^{* * *}$ & $-.24^{* *}$ & $-.28^{* * *}$ & $.40^{* * *}$ & $.29^{* * *}$ & $.16^{*}$ & $.48^{* * *}$ & 1 & & & & & \\
\hline & \multicolumn{18}{|c|}{ Scales relating to collaborative car use in general } \\
\hline 10 & Intention & 2.10 & 1.01 & .74 & $-.22^{* *}$ & $-.15^{*}$ & -.04 & $-.39^{* * *}$ & $.34^{* * *}$ & $.39^{* * *}$ & $.32^{* * *}$ & $.35^{* * *}$ & $.27^{* * *}$ & 1 & & & & \\
\hline 11 & $\begin{array}{c}\text { Perceived } \\
\text { Behaviour Control }\end{array}$ & 1.92 & 0.92 & .62 & $-.20^{* *}$ & -.08 & $-.17^{*}$ & $-.32^{* * *}$ & .12 & $.25^{* *}$ & $.23^{* *}$ & $.17^{*}$ & .12 & $.40^{* * *}$ & 1 & & & \\
\hline 12 & Social Norm & 1.94 & 1.01 & .85 & $-.20^{* *}$ & .05 & -.11 & $-.29^{* * *}$ & $.30^{* * *}$ & $.40^{* * *}$ & $.34^{* * *}$ & $.36^{* * *}$ & .13 & $.61^{* * *}$ & $.40^{* * *}$ & 1 & & \\
\hline 13 & Attitude & 2.28 & 1.07 & .80 & $-.20^{* *}$ & -.10 & -.13 & $-.49^{* * *}$ & $.28^{* * *}$ & $.35^{* * *}$ & $.30^{* * *}$ & $.42^{* * *}$ & $.27^{* * *}$ & $.59^{* * *}$ & $.51^{* * *}$ & $.47^{* * *}$ & 1 & \\
\hline 14 & Personal Norm & 2.32 & 1.17 & .91 & $-.21^{* *}$ & -.05 & -.06 & $-.23^{* *}$ & $.30^{* * *}$ & $.32^{* * *}$ & $.26^{* *}$ & $.32^{* * *}$ & $.17^{*}$ & $.64^{* * *}$ & $.25^{* * *}$ & $.64^{* * *}$ & $.39^{* * *}$ & 1 \\
\hline 15 & $\begin{array}{l}\text { Motorization } \\
\text { degree }\end{array}$ & 0.81 & 0.31 & - & -.14 & .02 & .03 & .10 & .11 & .01 & -.07 & .06 & .08 & .05 & $-.16^{*}$ & -.09 & -.05 & -.04 \\
\hline
\end{tabular}

Note. Variables 10-15 refer to collaborative mobility in general. Listwise case exclusion was used. Sample Size varies between $\mathrm{N}=158-172$ 
AUTONOMY LOSS, PRIVACY INVASION, AND DATA MISUSE IN COLLABORATIVE CAR USE

\section{Table C6}

Ridesharing: Descriptive Data and Intercorrelations of the Scales (Student Sample)

\begin{tabular}{|c|c|c|c|c|c|c|c|c|c|c|c|c|c|c|c|c|c|c|}
\hline & & $\mathrm{M}$ & $S D$ & $\alpha$ & 1 & 2 & 3 & 4 & 5 & 6 & 7 & 8 & 9 & 10 & 11 & 12 & 13 & 14 \\
\hline 1 & Privacy Invasion & 3.11 & 1.14 & .81 & 1 & & & & & & & & & & & & & \\
\hline 2 & Data Misuse & 3.03 & 1.20 & .77 & $.45^{* * *}$ & 1 & & & & & & & & & & & & \\
\hline 3 & $\begin{array}{l}\text { Interpersonal } \\
\text { Distrust }\end{array}$ & 2.78 & 1.20 & .81 & $.76^{* * *}$ & $.47^{* * *}$ & 1 & & & & & & & & & & & \\
\hline 4 & Autonomy Loss & 3.00 & 0.86 & .74 & $.47^{* * *}$ & $.36^{* *}$ & $.41^{* * *}$ & 1 & & & & & & & & & & \\
\hline 5 & $\begin{array}{c}\text { Perceived } \\
\text { Financial Benefit }\end{array}$ & 3.22 & 1.25 & - & $-.12^{*}$ & -.09 & -.08 & $-.24^{* * *}$ & 1 & & & & & & & & & \\
\hline 6 & $\begin{array}{c}\text { Intention } \\
\text { (Dichotomized }\end{array}$ & - & - & - & $-.13^{*}$ & -.11 & -.03 & $-.26^{* * *}$ & $-.31^{* * *}$ & 1 & & & & & & & & \\
\hline 7 & $\begin{array}{c}\text { Behaviour } \\
\text { (Dichotomized) }\end{array}$ & - & - & - & $-.15^{*}$ & -.03 & -.05 & $-.25^{* * *}$ & $.20^{* *}$ & $.23^{* * *}$ & 1 & & & & & & & \\
\hline 8 & $\begin{array}{l}\text { Neighbourhood } \\
\text { Scenario }\end{array}$ & 3.11 & 1.06 & - & $-.17^{* *}$ & -.02 & -.10 & $-.17^{* * *}$ & $.24^{* * *}$ & $.23^{* * *}$ & .06 & 1 & & & & & & \\
\hline 9 & Internet Scenario & 3.00 & 1.12 & - & $-.47^{* * *}$ & $-.29^{* * *}$ & $-.38^{* * *}$ & $-.31^{* * *}$ & $.24^{* * *}$ & $.22^{* * *}$ & .10 & $.23^{* * *}$ & 1 & & & & & \\
\hline \multicolumn{19}{|c|}{ Scales relating to collaborative car use in general } \\
\hline 10 & Intention & 2.81 & 1.01 & .87 & $-.19^{* *}$ & $-.19^{* *}$ & $-.12^{*}$ & $-.29^{* * *}$ & $.24^{* * *}$ & $.33^{* *}$ & $.30^{* * *}$ & $.20^{* *}$ & $.32^{* * *}$ & 1 & & & & \\
\hline 11 & $\begin{array}{c}\text { Perceived } \\
\text { Behaviour Control }\end{array}$ & 2.31 & 0.97 & .77 & $-.15^{*}$ & -.06 & -.05 & $-.33^{* * *}$ & $.17^{* *}$ & $.21^{* *}$ & $.26^{* * *}$ & .09 & $.19^{* *}$ & $.34^{* * *}$ & 1 & & & \\
\hline 12 & Social Norm & 2.34 & 1.00 & .82 & $-.13^{*}$ & $-.20^{* *}$ & -.07 & $-.13^{*}$ & $.26^{* * *}$ & $.29^{* * *}$ & $.17^{* * *}$ & $.17^{* *}$ & $.24^{* * *}$ & $.52^{* * *}$ & $.24^{* * *}$ & 1 & & \\
\hline 13 & Attitude & 3.00 & 0.99 & .72 & $-.30^{* * *}$ & $-.23^{* * *}$ & $-.19^{* *}$ & $-.49^{* * *}$ & $.29^{* * *}$ & $.28^{* * *}$ & $.31^{* * *}$ & $.20^{* *}$ & $.32^{* * *}$ & $.54^{* * *}$ & $.47^{* * *}$ & $.37^{* * *}$ & 1 & \\
\hline 14 & Personal Norm & 2.83 & 1.22 & .93 & $-.19^{* *}$ & $-.24^{* * *}$ & -.11 & $-.29^{* * *}$ & $.15^{*}$ & $.33^{* * *}$ & $.20^{* *}$ & $.19^{* *}$ & $.34^{* * *}$ & $.77^{* * *}$ & $.24^{* * *}$ & $.63^{* * *}$ & $.45^{* * *}$ & 1 \\
\hline 15 & $\begin{array}{c}\text { Motorization } \\
\text { degree }\end{array}$ & 0.52 & 0.41 & - & $-.16^{* *}$ & $.18^{* *}$ & $.16^{* *}$ & .08 & .11 & .08 & .12 & .05 & .01 & .00 & -.09 & .02 & -.05 & .03 \\
\hline
\end{tabular}

Note. Variables 10-15 refer to collaborative mobility in general. Listwise case exclusion was used. Sample Size varies between $\mathrm{N}=256-265$ 